Article

\title{
Euler-Bernoulli Beam Theory: First-Order Analysis, Second-Order Analysis, Stability, and Vibration Analysis Using the Finite Difference Method
}

\author{
Valentin Fogang \\ Civil Engineer, c/o BUNS Sarl, P.O Box 1130, Yaounde, Cameroon; valentin.fogang@bunscameroun.com
}

ORCID iD https://orcid.org/0000-0003-1256-9862

\begin{abstract}
This paper presents an approach to the Euler-Bernoulli beam theory (EBBT) using the finite difference method (FDM). The EBBT covers the case of small deflections, and shear deformations are not considered. The FDM is an approximate method for solving problems described with differential equations. The FDM does not involve solving differential equations; equations are formulated with values at selected points of the structure. Generally, the finite difference approximations are derived based on fourth-order polynomial hypothesis (FOPH) and second-order polynomial hypothesis (SOPH) for the deflection curve; the FOPH is made for the fourth and third derivative of the deflection curve while the SOPH is made for its second and first derivative. In addition, the boundary conditions and not the governing equations are applied at the beam's ends. In this paper, the FOPH was made for all of the derivatives of the deflection curve, and additional points were introduced at the beam's ends and positions of discontinuity (concentrated loads or moments, supports, hinges, springs, etc.). The introduction of additional points allowed us to apply the governing equations at the beam's ends and to satisfy the boundary and continuity conditions. Moreover, grid points with variable spacing were also considered, the grid being uniform within beam segments. Firstorder analysis, second-order analysis, and vibration analysis of structures were conducted with this model.

Furthermore, tapered beams were analyzed (element stiffness matrix, second-order analysis). Finally, a direct time integration method (DTIM) was presented. The FDM-based DTIM enabled the analysis of forced vibration of structures, with damping taken into account. The results obtained in this paper showed good agreement with those of other studies, and the accuracy was increased through a grid refinement. Especially in the first-order analysis of uniform beams, the results were exact for uniformly distributed and concentrated loads regardless of the grid. Further research will be needed to investigate polynomial refinements (higher-order polynomials such as fifth-order, sixthorder...) of the deflection curve; the polynomial refinements aimed to increase the accuracy, whereby non-centered finite difference approximations at beam's ends and positions of discontinuity would be used.
\end{abstract}

Keywords: Euler-Bernoulli beam; finite difference method; additional points; element stiffness matrix; tapered beam; second-order analysis; vibration analysis; boundary value problem; direct time integration method 


\section{EULER-BERNOULLI BEAM THEORY USING THE FINITE DIFFERENCE METHOD}

\section{Introduction}

The Euler-Bernoulli beam has been widely analyzed in the literature. Several methods have been developed, such as the force method, the slope deflection method, and the direct stiffness method. The analytical approach consists of solving the governing equations (i.e., statics and material) that are expressed via means of differential equations, and satisfying the boundary and continuity conditions. However, solving the differential equations may be difficult in the presence of an axial force (or external distributed axial forces), an elastic Winkler foundation, tapered beams, or damping (by vibration analysis). Numerical methods permit therefore to overcome solving the differential equations. A considerable volume of literature has been published on numerical methods for Euler-Bernoulli beam analysis. Anley et al. [1] considered a numerical difference approximation for solving twodimensional Riesz space fractional convection-diffusion problem with source term over a finite domain. Kindelan et al. [2] presented a method to obtain optimal finite difference formulas which maximize their frequency range of validity. Both conventional and staggered equispaced stencils for first and second derivatives were considered. Torabi et al. [3] presented an exact closed-form solution for free vibration analysis of Euler-Bernoulli conical and tapered beams carrying any desired number of attached masses; the concentrated masses were modeled by Dirac's delta functions. Katsikadelis [4] presented a direct time integration method for the solution of the equations of motion describing the dynamic response of structural linear and nonlinear multi-degree-of-freedom systems. The method applied also to equations with variable coefficients. Soltani et al. [5] applied the Finite Difference Method (FDM) to evaluate natural frequencies of non-prismatic beams, with different boundary conditions and resting on variable one or two parameter elastic foundations. Boreyri et al. [6] analyzed the free vibration of a new type of tapered beam, with exponentially varying thickness, resting on a linear foundation. The solution was based on a semi-analytical technique, the differential transform method. Mwabora et al. [7] considered numerical solutions for static and dynamic stability parameters of an axially loaded uniform beam resting on a simply supported foundations using FDM where Central Difference Scheme was developed. In the classical beam analysis using the FDM, the finite difference approximations are derived based on fourth order polynomial hypothesis (FOPH) and second-order polynomial hypothesis (SOPH) for the deflection curve; the FOPH is made for the fourth and third derivative of the deflection curve while the SOPH is made for the second and first derivative. In addition, points outside the beam are generally not considered; the boundary conditions are applied at the beam's ends and not the governing equations. Consequently, the non-application of the governing equations at the beam's ends together with the different polynomial hypotheses for the deflection curve have led to inaccurate results, making the FDM less interesting in comparison to other numerical methods such as the finite element method. In this paper, a model based on FDM was presented. This model consisted of formulating the differential equations with finite differences and introducing additional points at beam's ends and at positions of discontinuity (concentrated loads or moments, supports, hinges, springs, change of grid spacing, and brutal change of stiffness). The introduction of additional points allowed us to apply the governing equations at the beam's ends and to satisfy the boundary and continuity conditions. Furthermore, the finite difference approximations were derived using the FOPH for all of the derivatives of the deflection curve. Finally, a direct time integration method (DTIM) was presented; the FDM-based DTIM enabled the analysis of forced vibration of structures, the damping being considered. 


\section{Materials and methods}

\subsection{First-order analysis}

The sign conventions adopted for loads, bending moments, shear forces, and displacements are illustrated in Figure 1.

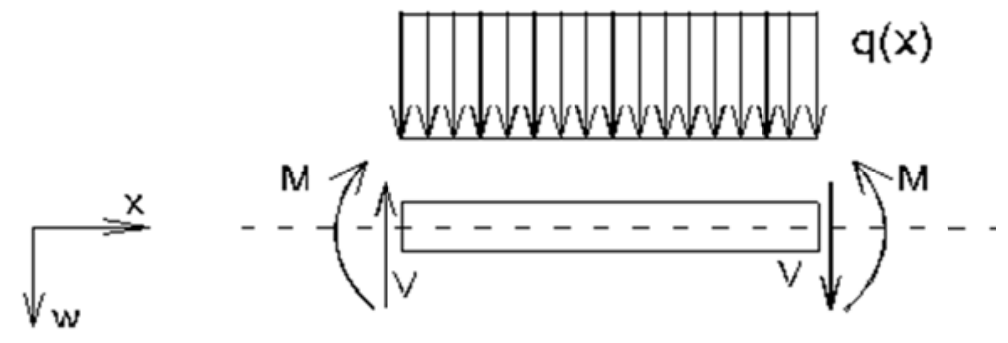

Figure 1 Sign convention for loads, bending moments, shear forces, and displacements

Specifically, $\mathrm{M}(\mathrm{x})$ is the bending moment in the section, $\mathrm{V}(\mathrm{x})$ is the shear force, $\mathrm{w}(\mathrm{x})$ is the deflection, and $\mathrm{q}(\mathrm{x})$ is the distributed load in the positive downward direction.

\subsubsection{First-order analysis of uniform beam within segments}

\subsubsection{Statics}

According to Euler-Bernoulli beam theory (EBBT), the governing equation of a beam loaded with $\mathrm{q}(\mathrm{x})$ is as follows:

$$
E I \frac{d^{4} w(x)}{d x^{4}}+k(x) w(x)=q(x),
$$

where $\mathrm{EI}$ is the flexural stiffness and $\mathrm{k}(\mathrm{x})$ is the stiffness of the elastic Winkler foundation. The bending moment, shear force, and slope $\varphi(\mathrm{x})$ are related to the deflection as follows:

$$
\begin{aligned}
& M(x)=-E I \frac{d^{2} w(x)}{d x^{2}} \\
& V(x)=\frac{d M(x)}{d x} \rightarrow V(x)=-E I \frac{d^{3} w(x)}{d x^{3}} \\
& \varphi(x)=\frac{d w(x)}{d x}
\end{aligned}
$$

\subsubsection{Fundamentals of FDM for a uniform beam}

Figure 2 shows a segment of a beam having equidistant points with grid spacing h.

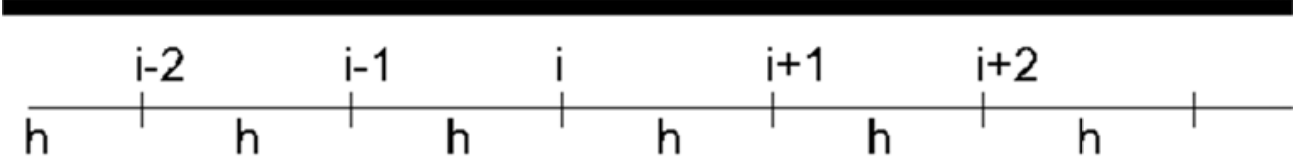

Figure 2 Beam with equidistant points 
The governing equation (Equation (1)) has a fourth order derivative, the deflection curve is consequently approximated around the point of interest $i$ as a fourth degree polynomial.

The deflection curve can be described with the values of deflections at equidistant grid points:

$$
w(x)=w_{i-2} \times f_{i-2}(x)+w_{i-1} \times f_{i-1}(x)+w_{i} \times f_{i}(x)+w_{i+1} \times f_{i+1}(x)+w_{i+2} \times f_{i+2}(x)
$$

The shape functions $\mathrm{f}_{\mathrm{j}}(\mathrm{x})(\mathrm{j}=\mathrm{i}-2 ; \mathrm{i}-1 ; \mathrm{i} ; \mathrm{i}+1 ; \mathrm{i}+2)$ can be expressed using the Lagrange polynomials:

$$
f_{j}(x)=\prod_{\substack{k=i-2 \\ k \neq j}}^{i+2} \frac{x-x_{k}}{x_{j}-x_{k}}
$$

Thus, a five-point stencil is used to derive finite difference approximations to derivatives at grid points. The derivatives at $\mathbf{i}$ are expressed with values of deflection at points i-2; i-1; i; i+1; i+2.

$$
\begin{aligned}
& \left.\frac{d^{4} w}{d x^{4}}\right|_{i}=\frac{w_{i-2}-4 w_{i-1}+6 w_{i}-4 w_{i+1}+w_{i+2}}{h^{4}} \\
& \left.\frac{d^{3} w}{d x^{3}}\right|_{i}=\frac{-w_{i-2}+2 w_{i-1}-2 w_{i+1}+w_{i+2}}{2 h^{3}} \\
& \left.\frac{d^{2} w}{d x^{2}}\right|_{i}=\frac{-w_{i-2}+16 w_{i-1}-30 w_{i}+16 w_{i+1}-w_{i+2}}{12 h^{2}} \\
& \left.\frac{d w}{d x}\right|_{i}=\frac{w_{i-2}-8 w_{i-1}+8 w_{i+1}-w_{i+2}}{12 h}
\end{aligned}
$$

\subsubsection{FDM Formulation of equations, efforts, and deformations}

Let us consider a segment $\mathrm{k}$ of the beam having a flexural stiffness $\mathrm{EI}_{\mathrm{k}}$ and equidistant grid points with spacing $\mathrm{h}_{\mathrm{k}}$. We introduce a reference flexural stiffness $\mathrm{EI}_{\mathrm{r}}$ as follows

$$
\mathrm{EI}_{\mathrm{k}}=\beta_{\mathrm{k}} \times \mathrm{EI}_{\mathrm{r}}
$$

We set

$$
\mathrm{W}(\mathrm{x})=\mathrm{EI}_{\mathrm{r}} \times \mathrm{W}(\mathrm{x})
$$

Substituting Equations (4a), (5), and (5a) into Equation (1) yields

$$
W_{i-2}-4 W_{i-1}+\left(6+\frac{k_{i} h_{k}^{4}}{\beta_{k} E I_{r}}\right) W_{i}-4 W_{i+1}+W_{i+2}=\frac{1}{\beta_{k}} q_{i} h_{k}^{4}
$$


At point $\mathrm{i}$, the bending moment, shear force, and slope are formulated with finite differences using Equations (2a-c), (4b-d), (5), and (5a).

$$
\begin{aligned}
& M_{i}=\beta_{k} \frac{W_{i-2}-16 W_{i-1}+30 W_{i}-16 W_{i+1}+W_{i+2}}{12 h_{k}^{2}} \\
& V_{i}=\beta_{k} \frac{W_{i-2}-2 W_{i-1}+2 W_{i+1}-W_{i+2}}{2 h_{k}^{3}} \\
& E I_{r} \varphi_{i}=\frac{W_{i-2}-8 W_{i-1}+8 W_{i+1}-W_{i+2}}{12 h_{k}}
\end{aligned}
$$

\subsubsection{FDM Formulation of loadings}

Let us determine here the FDM value $\mathrm{q}_{\mathrm{i}}$ (Equation (6)) in the case of a varying distributed load $\mathrm{q}(\mathrm{x})$. Without considering the elastic Winkler foundation the distributed load $\mathrm{q}(\mathrm{x})$ is related to the shear force $\mathrm{V}(\mathrm{x})$ as follows:

$$
q(x)=-\frac{d V(x)}{d x}
$$

Considering here a three-point stencil, the following FDM formulations of the first derivative can be made. The position $\mathrm{i}$ is considered as the left beam's end, an interior point on the beam, or the right beam's end, respectively:

$$
\begin{aligned}
& \left.\frac{d V(x)}{d x}\right|_{i}=\frac{-3 V_{i}+4 V_{i+1}-V_{i+2}}{2 h} \\
& \left.\frac{d V(x)}{d x}\right|_{i}=\frac{-V_{i-1}+V_{i+1}}{2 h} \\
& \left.\frac{d V(x)}{d x}\right|_{i}=\frac{V_{i-2}-4 V_{i-1}+3 V_{i}}{2 h}
\end{aligned}
$$

The balance of vertical forces applied to a free body diagrams yields the following:

$$
\begin{aligned}
& V_{i}-V_{i-1}=-\int_{i-1}^{i} q(x) d x \\
& V_{i+1}-V_{i}=-\int_{i}^{i+1} q(x) d x
\end{aligned}
$$

The combination of Equations ( $8 \mathrm{a}-\mathrm{f}$ ) yields the FDM value $\mathrm{q}_{\mathrm{i}}$ for the position i being the left beam's end, an interior point on the beam, or the right beam's end.

$$
\begin{aligned}
& q_{i}=\frac{1}{2 h}\left[3 \int_{i}^{i+1} q(x) d x-\int_{i+1}^{i+2} q(x) d x\right] \\
& q_{i}=\frac{1}{2 h} \int_{i-1}^{i+1} q(x) d x \\
& q_{i}=\frac{1}{2 h}\left[-\int_{i-2}^{i-1} q(x) d x+3 \int_{i-1}^{i} q(x) d x\right]
\end{aligned}
$$


The application of Equations (8g-8i) shows that in the case of a linearly distributed load, $\mathrm{q}_{\mathrm{i}}$ is equal $\mathrm{q}\left(\mathrm{x}_{\mathrm{i}}\right)$.

At point $\mathrm{i}$, the stiffness of the elastic Winkler foundation ki is calculated similarly to Equations (8g-8i).

\subsubsection{Analysis at positions of discontinuity}

Positions of discontinuity are positions of application of concentrated external loads (force or moment), supports, hinges, springs, abrupt change of cross section, and change of grid spacing.

\subsection{Equations of continuity}

Let us consider segments $\mathrm{k}$ and $\mathrm{p}$ of the beam having flexural stiffness $\mathrm{EI}_{\mathrm{k}}$ and $\mathrm{EI}_{\mathrm{p}}$, and equidistant grid points with spacing $h_{k}$ and $h_{p}$. Concentrated loads (force $\mathrm{P}$ and moment $\mathrm{M}^{*}$ ) are applied at point $\mathrm{i}$, as represented in Figure 3 .

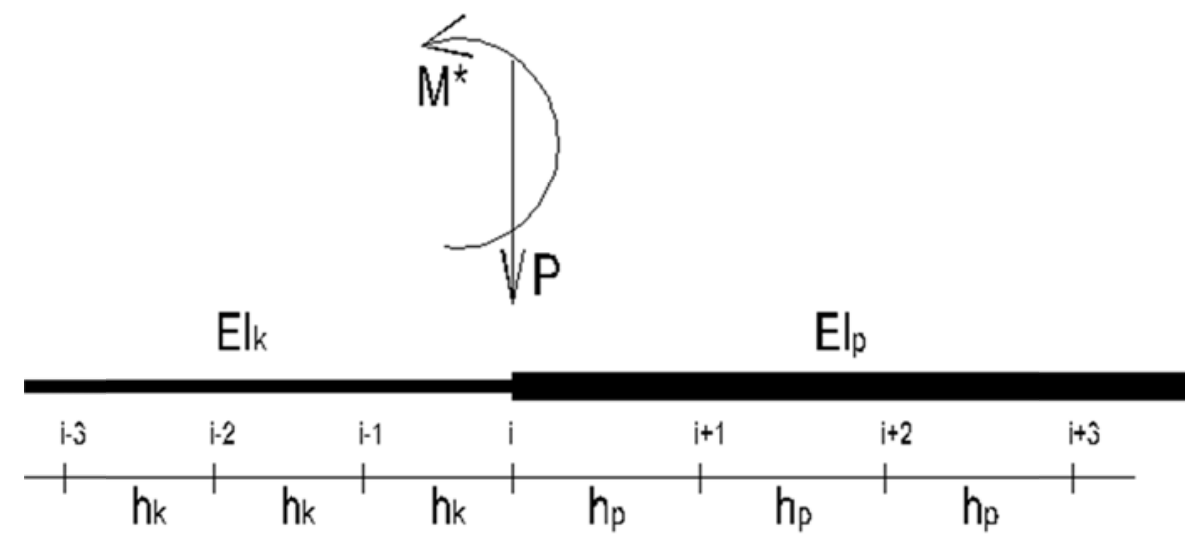

Figure 3 Beam with change in grid spacing and stiffness

The model developed in this paper consists of realizing an opening of the beam at point $\mathrm{i}$ and introducing additional points (fictive points ia, ib, ic, and id) in the opening, as represented in Figure 4a,b.

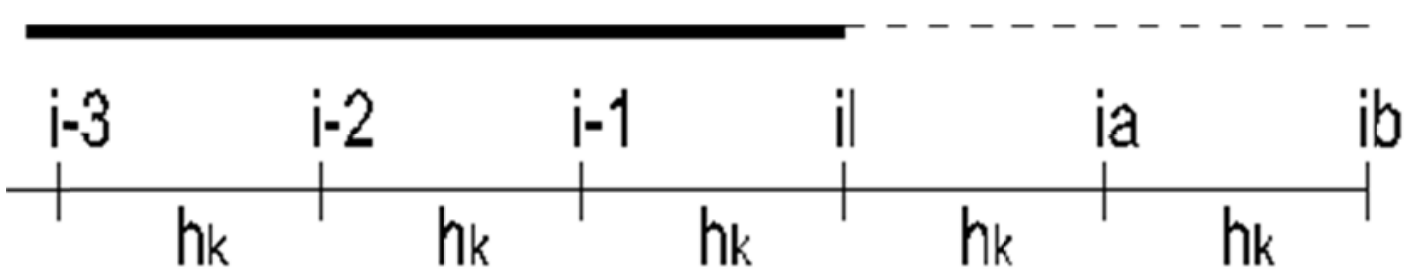

(4a)

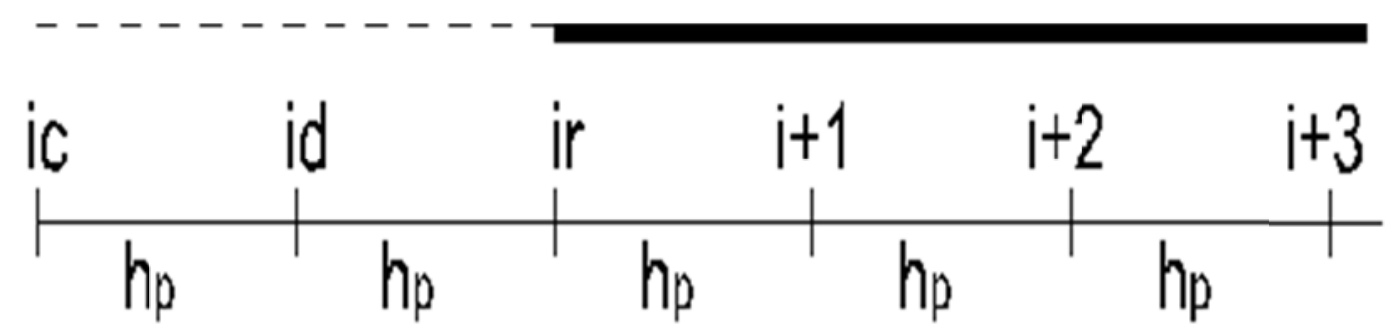

Figure 4a,b Opening of the beam and introduction of additional points on the left side (4a) and right side (4b) 
The governing equation (Equation (6)) is applied at any point of the beam, i.e., i-2; i-1; il; ir; i+1; i+2, etc. Thus, the governing equations at positions il and ir yield:

$$
\begin{aligned}
& W_{i-2}-4 W_{i-1}+\left(6+\frac{k_{i l} h_{k}^{4}}{\beta_{k} E I_{r}}\right) W_{i l}-4 W_{i a}+W_{i b}=\frac{1}{\beta_{k}} q_{i l} h_{k}^{4} \\
& W_{i c}-4 W_{i d}+\left(6+\frac{k_{i r} h_{p}^{4}}{\beta_{p} E I_{r}}\right) W_{i r}-4 W_{i+1}+W_{i+2}=\frac{1}{\beta_{p}} q_{i r} h_{p}^{4}
\end{aligned}
$$

The FDM formulations $\mathrm{q}_{\mathrm{il}}$ and $\mathrm{q}_{\mathrm{ir}}$ of distributed loading and $\mathrm{k}_{\mathrm{il}}$ and $\mathrm{k}_{\mathrm{ir}}$ of elastic Winkler foundation are calculated using Equations (8g-i).

The following continuity equations express the continuity of the deflection and slope (Equation (7c)), and the equilibrium of the bending moment (Equation (7a)) and shear force (Equation (7b)):

$$
\begin{aligned}
& W_{i l}=W_{i r} \rightarrow W_{i l}=W_{i r} \\
& E I_{r} \varphi_{i l}=E I_{r} \varphi_{i r} \rightarrow \frac{W_{i-2}-8 W_{i-1}+8 W_{i a}-W_{i b}}{12 h_{k}}=\frac{W_{i c}-8 W_{i d}+8 W_{i+1}-W_{i+2}}{12 h_{p}} \\
& M_{i l}-M_{i r}=M^{*} \rightarrow \\
& \beta_{k} \frac{W_{i-2}-16 W_{i-1}+30 W_{i l}-16 W_{i a}+W_{i b}}{12 h_{k}{ }^{2}}-\beta_{p} \frac{W_{i c}-16 W_{i d}+30 W_{i r}-16 W_{i+1}+W_{i+2}}{12 h_{p}{ }^{2}}=M^{*} \\
& V_{i l}-V_{i r}=P \rightarrow \beta_{k} \frac{W_{i-2}-2 W_{i-1}+2 W_{i a}-W_{i b}}{2 h_{k}{ }^{3}}-\beta_{p} \frac{W_{i c}-2 W_{i d}+2 W_{i+1}-W_{i+2}}{2 h_{p}{ }^{3}}=P
\end{aligned}
$$

An adjustment of the continuity equations is made in case of a hinge (no continuity of the slope, $M_{i l}=M_{i r}=0$ ), a support $\left(\mathrm{W}_{\mathrm{il}}=\mathrm{W}_{\mathrm{ir}}=0\right.$, no equation (10d)), or a spring.

At the beam's ends, additional points are introduced (as shown in Figure 4a,b) and so governing equations are applied at the beam's ends, as well as the boundary conditions.

\subsection{Change of grid spacing}

The discretization of the beam may lead to uniform-grid segments, but the grid spacings being different from one segment to another, as represented in Figure 5.

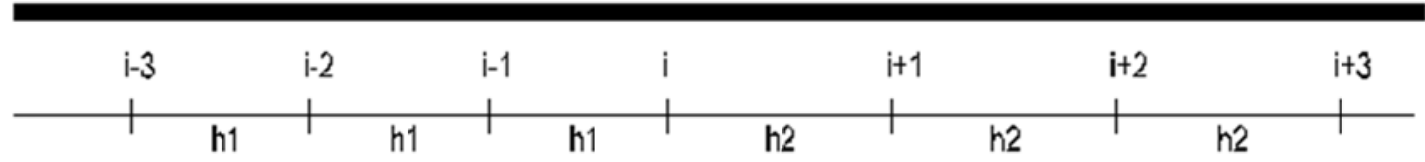

Figure 5 Beam with different grid spacings 
The governing equation (Equation (6)), and the equations for the determination of the bending moment, shear force, and slope (Equations (7a-c)) at position $\mathbf{i}$ are formulated under consideration of differentt grid spacings h1 and h2. The continuity equations (Equations (10a-b) and (11-12) also apply.

\subsection{Non-uniform grid}

The grid may be such that every node has a non-constant distance from another, as represented in Figure 6 .

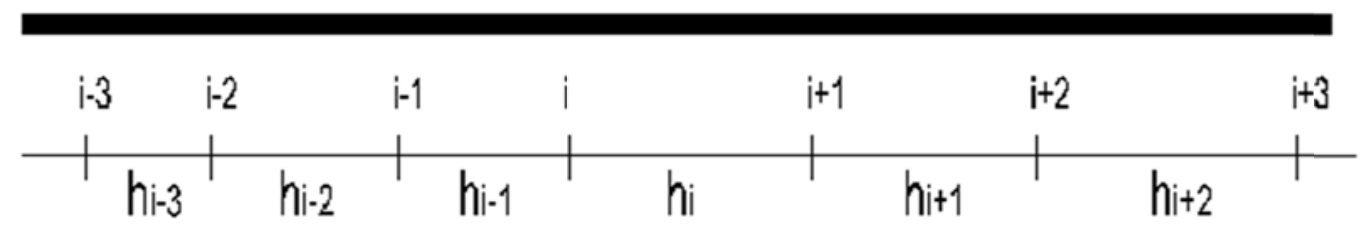

Figure 6 Beam with a non-uniform grid

Here, the Lagrange interpolation polynomial (Equation (3b)) is used for FDM formulation. The resulting equations are complicated, and consequently the non-uniform grid is not further analyzed in this paper. In fact, this case should not be analyzed as a discontinuity position.

\subsubsection{First-order analysis of a tapered beam}

\subsubsection{Statics}

The case of a tapered beam, as shown in Figure 7, was analyzed.

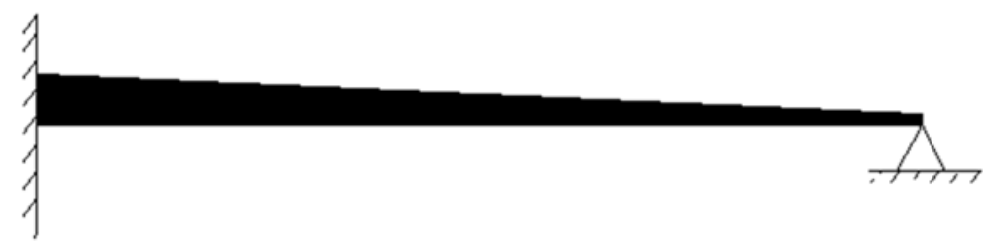

Figure 7 Tapered beam

The stiffness of the elastic Winkler foundation is denoted by $\mathrm{k}(\mathrm{x})$ is considered. The varying flexural stiffness is $\mathrm{EI}(\mathrm{x})$. The equations of static equilibrium and material relation are formulated as follows:

$$
\begin{aligned}
& \frac{d^{2} M(x)}{d x^{2}}-k(x) w(x)=-q(x) \\
& M(x)=-E I(x) \frac{d^{2} w(x)}{d x^{2}}
\end{aligned}
$$

The slope is determined using Equation (2c), and the shear force using (2b). 


\subsubsection{FDM formulations of equations, efforts, and deformations}

Two FDM approximations were considered for the analysis of tapered beams: the M-W and the W FDM approximation.

M-W FDM approximation: The unknowns at a given point are the deflection and the bending moment. A uniform grid with spacing $h_{k}$ is considered. Equations (13a-b) have a second order derivative; consequently, a three-point stencil is considered for the following derivatives $(S(x)$ representing $M(x)$ or $w(x))$ :

$$
\begin{aligned}
& \left.\frac{d^{2} S(x)}{d x^{2}}\right|_{i}=\frac{S_{i-1}-2 S_{i}+S_{i+1}}{h_{k}^{2}} \\
& \left.\frac{d S(x)}{d x}\right|_{i}=\frac{-S_{i-1}+S_{i+1}}{2 h_{k}}
\end{aligned}
$$

A reference flexural rigidity $E I_{r}$ is introduced (Equations (5-5a)). Here the parameter $\beta$ is defined at any position $i$. Considering Equations (5-5a) and (14a), the FDM formulations of Equations (13a,b) yield

$$
\begin{aligned}
& \frac{M_{i-1}-2 M_{i}+M_{i+1}}{h_{k}^{2}}-k_{i} w_{i}=-q_{i} \rightarrow h_{k}{ }^{2} M_{i-1}-2 h_{k}^{2} M_{i}+h_{k}{ }^{2} M_{i+1}-\frac{k_{i} h_{k}^{4}}{E I_{r}} W_{i}=-q_{i} h_{k}^{4} \\
& M_{i}=-E I_{i} \frac{w_{i-1}-2 w_{i}+w_{i+1}}{h_{k}{ }^{2}} \rightarrow h_{k}{ }^{2} M_{i}+\beta_{i} W_{i-1}-2 \beta_{i} W_{i}+\beta_{i} W_{i+1}=0
\end{aligned}
$$

At any point on the grid, Equations (15a-b) are applied. The application of Equations (2b), (2c), and (14b) yields the shear force and slope:

$$
\begin{gathered}
V_{i}=\left.\frac{d M(x)}{d x}\right|_{i}=\frac{-M_{i-1}+M_{i+1}}{2 h_{k}} \\
\varphi_{i}=\left.\frac{d w(x)}{d x}\right|_{i}=\frac{-w_{i-1}+w_{i+1}}{2 h_{k}}
\end{gathered}
$$

W FDM approximation: The unknown at a given point is the deflection. Substituting Equation (13b) into (13a) yields

$$
-\frac{d^{2}}{d x^{2}}\left[E I(x) \frac{d^{2} w(x)}{d x^{2}}\right]-k(x) w(x)=-q(x)
$$

Developing Equation (15e) yields

$$
E I(x) \frac{d^{4} w(x)}{d x^{4}}+2 \frac{d[E I(x)]}{d x} \frac{d^{3} w(x)}{d x^{3}}+\frac{d^{2}[E I(x)]}{d x^{2}} \frac{d^{2} w(x)}{d x^{2}}+k(x) w(x)=q(x)
$$


Since the flexural stiffness EI(x) of the beam varies throughout the longitudinal axis, reference values are defined like for uniform beams (see Equation (5)). The reference length and the grid spacing are denoted by $l_{r}$.and $h_{k}$, respectively.

$$
I(x)=\beta_{I}(x) \times I_{r} \quad(15 g) \quad h_{k}=\beta_{l k} l_{r}
$$

The parameters $\beta_{\mathrm{I}}{ }_{\mathrm{I}}(x)$ and $\beta^{\prime \prime}{ }_{\mathrm{I}}(x)$ are related to the first and second derivative of $\beta_{\mathrm{I}}(x)$ with respect to $x$, respectively, as follows:

$$
\beta_{I}^{\prime}(x)=h_{k} \frac{d \beta_{I}(x)}{d x}
$$

$$
\beta_{I}^{\prime \prime}(x)=h_{k}^{2} \frac{d^{2} \beta_{I}(x)}{d x^{2}}
$$

Applying Equations (15g-j), (4a-c), and (5a) into Equation (15f) yields the following FDM formulation:

$$
\begin{aligned}
& {\left[\beta_{I i}-\beta_{I i}^{\prime}-\frac{\beta_{I i}^{\prime \prime}}{12}\right] W_{i-2}+\left[-4 \beta_{I i}+2 \beta_{I i}^{\prime}+\frac{4 \beta_{I i}^{\prime \prime}}{3}\right] W_{i-1}} \\
& {\left[6 \beta_{I i}-\frac{5 \beta_{I i}^{\prime \prime}}{2}+\frac{k_{i} h_{k}^{4}}{E_{r} I_{r}}\right] W_{i}+\left[-4 \beta_{I i}-2 \beta_{I i}^{\prime}+\frac{4 \beta_{I i}^{\prime \prime}}{3}\right] W_{i+1}+\left[\beta_{I i}+\beta_{I i}^{\prime}-\frac{\beta_{I i}^{\prime \prime}}{12}\right] W_{i-2}=q_{i} h_{k}^{4}}
\end{aligned}
$$

The rotation of the cross section is calculated using Equation (7c). The bending moment and the shear force are calculated using Equations (2a), (4c), (5a), and (15g), and Equations (2b), (4b-c), (5a), (15i), and (15g) as follows:

$$
\begin{aligned}
& M_{i}=\beta_{I i} \frac{W_{i-2}-16 W_{i-1}+30 W_{i}-16 W_{i+1}+W_{i+2}}{12 h_{k}^{2}} \\
& V_{i}=\beta_{I i}^{\prime} \frac{W_{i-2}-16 W_{i-1}+30 W_{i}-16 W_{i+1}+W_{i+2}}{12 h_{k}^{3}}+\beta_{I i} \frac{W_{i-2}-2 W_{i-1}+2 W_{i+1}-W_{i+2}}{2 h_{k}^{3}}
\end{aligned}
$$

\subsubsection{Analysis of a tapered beam at positions of discontinuity}

\section{M-W FDM approximation}

Concentrated loads (force $\mathrm{P}$ and moment $\mathrm{M}^{*}$ ) are applied at point $\mathrm{i}$ (see Figure 4). A change in grid spacing is also assumed at this position. As described in section 2.1.1.5, an opening of the beam at point $\mathrm{i}$ introduced additional points (points ia and id) in the opening, as represented in Figure 8a,b.

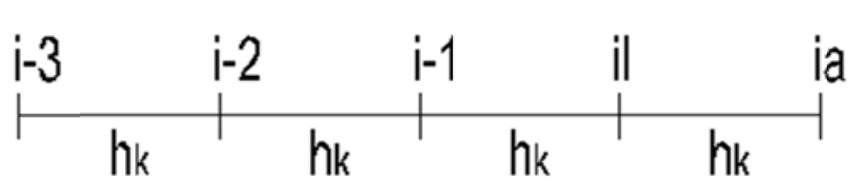




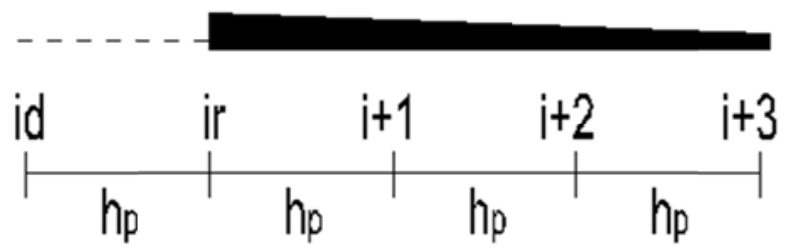

Figure 8a,b Opening of the beam and introduction of additional points at the left side (8a) and right side (8b)

The additional points are ia and id, and the unknowns are Wia, Mia, Wid, and Mid.

The continuity equations express the continuity of the deflection and slope (Equation (15d)), and the equilibrium of the bending moment and shear force (Equation (15c)) as follows:

$$
\begin{aligned}
& w_{i l}=w_{i r} \rightarrow W_{i l}=W_{i r} \\
& E I_{r} \varphi_{i l}=E I_{r} \varphi_{i r} \rightarrow \frac{-W_{i-1}+W_{i a}}{2 h_{k}}=\frac{-W_{i d}+W_{i+1}}{2 h_{p}} \\
& M_{i l}-M_{i r}=M^{*} \\
& V_{i l}-V_{i r}=P \rightarrow \frac{-M_{i-1}+M_{i a}}{2 h_{k}}-\frac{-M_{i d}+M_{i+1}}{2 h_{p}}=P
\end{aligned}
$$

At a point i with a hinge the slopes are not equal anymore, and the moments Mil and Mir are zero.

\section{W FDM approximation:}

Similarly to the M-W FDM approximation, an opening of the beam at point $\mathbf{i}$ introduced additional points (points ia, ib, ic, and id) in the opening, as represented in Figure 4a,b.

The continuity equations (Equations (16a-d)) are applied. However, the bending moment, the shear force, and the rotation of the cross section are calculated using Equations (15l), (15m), and (7c), respectively.

\section{Mixed FDM approximation}

M-W FDM approximation and W FDM approximation can be considered on either side of the point of discontinuity. This may be helpful when a uniform beam segment (W FDM approximation) and a tapered beam segment (M-W FDM approximation) are connected. The continuity equations are then formulated with the corresponding formulas.

\subsubsection{First-order element stiffness matrix of a tapered beam}

The M-W FDM approximation (Equations (15a-d)) is used in this section. Nevertheless, the W FDM approximation could also be considered with appropriate equations (Equations (15k) with $\mathrm{q}_{\mathrm{i}}=0$, (15l-m)). 


\subsubsection{1 $4 \times 4$ element stiffness matrix}

The sign conventions for bending moments, shear forces, displacements, and slope adopted for use in determining the element stiffness matrix in local coordinates is illustrated in Figure 9.

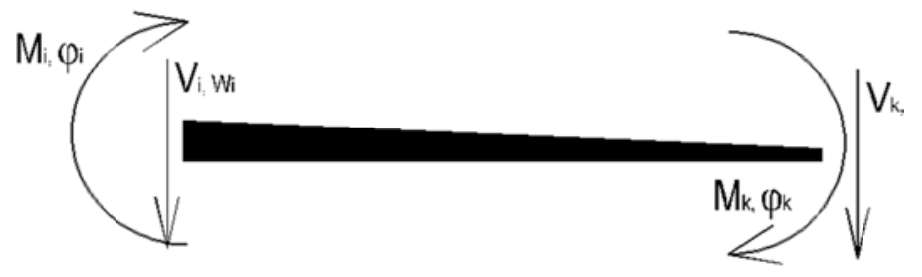

Figure 9 Sign conventions for moments, shear forces, displacements, and slopes for stiffness matrix

Let us define following vectors:

$$
\overrightarrow{S_{\text {red }}}=\left[V_{i} ; M_{i} ; V_{k} ; M_{k}\right]^{T} \quad(17 \mathrm{a}) \quad \overrightarrow{V_{\text {red }}}=\left[w_{i} ; \varphi_{i} ; w_{k} ; \varphi_{k}\right]^{T}
$$

The $4 \times 4$ element stiffness matrix in local coordinates of the tapered beam is denoted by $\mathrm{K}_{44}$.

The vectors above are related together with the element stiffness matrix $\mathrm{K}_{44}$ as follows:

$$
\overrightarrow{S_{\text {red }}}=K_{44} \times \overrightarrow{V_{\text {red }}}
$$

Let us divide the beam in $\mathrm{n}$ parts of equal length $\mathrm{h}(\mathrm{l}=\mathrm{nh})$ as shown in Figure 10.
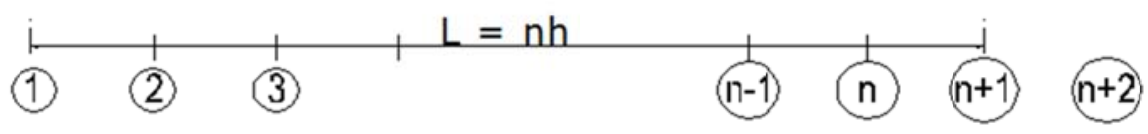

Figure 10 Finite difference method (FDM) discretization for $4 \times 4$ element stiffness matrix

Equations (15a) with $\mathrm{q}_{\mathrm{i}}=0$ and (15b) are applied at any point on the grid (positions $1 ; 2 ; \ldots \mathrm{n}+1$ of Figure 10).

Considering the sign conventions adopted for bending moments and shear forces in general (see Figure 1) and for bending moments and shear forces in the element stiffness matrix (see Figure 9), we can set following static compatibility boundary conditions in combination with Equations (2b) and (14b):

$$
\begin{aligned}
& V_{i}=-V_{1}=-\left.\frac{d M(x)}{d x}\right|_{1}=-\frac{M_{2}-M_{0}}{2 h} \rightarrow 2 h V_{i}+M_{2}-M_{0}=0 \\
& M_{i}=M_{1} \rightarrow M_{i}-M_{1}=0 \\
& V_{k}=V_{n+1}=\left.\frac{d M(x)}{d x}\right|_{n+1}=\frac{M_{n+2}-M_{n}}{2 h} \rightarrow 2 h V_{k}-M_{n+2}+M_{n}=0 \\
& M_{k}=-M_{n+1} \rightarrow M_{k}+M_{n+1}=0
\end{aligned}
$$


Considering the sign conventions adopted for the displacements and slope in general (see Figure 1) and for displacements and slope in the element stiffness matrix (see Figure 9), we can set following geometric compatibility boundary conditions in combination with Equations (2c) and (14b):

$$
\begin{aligned}
& w_{1}=w_{i} \rightarrow W_{1}=E I_{r} \times w_{i} \\
& \varphi_{1}=\left.\frac{d w(x)}{d x}\right|_{1}=\frac{-w_{0}+w_{2}}{2 h}=\varphi_{i} \rightarrow \frac{-W_{0}+W_{2}}{2 h}=E I_{r} \times \varphi_{i} \\
& w_{n+1}=w_{k} \rightarrow W_{n+1}=E I_{r} \times w_{k} \\
& \varphi_{n+1}=\left.\frac{d w(x)}{d x}\right|_{n+1}=\frac{-w_{n}+w_{n+2}}{2 h}=\varphi_{k} \rightarrow \frac{-W_{n}+W_{n+2}}{2 h}=E I_{r} \times \varphi_{k}
\end{aligned}
$$

The number of equations is $2(n+1)+4+4=\mathbf{2 n}+\mathbf{1 0}$. The number of unknowns is $2(n+3)+4=\mathbf{2 n}+\mathbf{1 0}$, especially 2(n+3) unknowns (M; W) at points on the beam and additional points at beam's ends, and 4 efforts at beam's ends ( $\mathrm{V}_{\mathrm{i}}$; $\mathrm{M}_{\mathrm{i}} ; \mathrm{V}_{\mathrm{k}} ; \mathrm{M}_{\mathrm{k}}$ ). Let us define following vector

$$
\vec{S}_{1}=\left[M_{0} ; W_{0} ; M_{1} ; W_{1} \ldots \ldots M_{n+2} ; W_{n+2}\right]^{T}
$$

The combination of Equations (15a,b) applied at any point on the grid, Equations (19a-d), and Equations (20a-d) can be expressed with matrix notation as follows, the geometric compatibility boundary conditions (Equations (20a-d)) being at the bottom.

$$
T \times\left[\begin{array}{c}
\vec{S}_{1} \\
\vec{S}_{\text {red }}
\end{array}\right]=\left[\begin{array}{c}
\overrightarrow{0} \\
E I_{r} \times \vec{V}_{\text {red }}
\end{array}\right] \rightarrow\left[\begin{array}{c}
\vec{S}_{1} \\
\vec{S}_{r e d}
\end{array}\right]=T^{-1} \times\left[\begin{array}{c}
\overrightarrow{0} \\
E I_{r} \times \vec{V}_{\text {red }}
\end{array}\right]
$$

The matrix $\mathbf{T}$ has $2 \mathrm{n}+10$ rows and $2 \mathrm{n}+10$ columns. The zero vector above has $2 \mathrm{n}+6$ rows.

$$
T^{-1}=\left[\begin{array}{ll}
T_{a a} & T_{a b} \\
T_{b a} & T_{b b}
\end{array}\right]
$$

The matrix Taa has $2 n+6$ rows and $2 n+6$ columns, the matrix Tab has $2 n+6$ rows and 4 columns, the matrix Tba has 4 rows and $2 n+6$ columns, and the matrix Tbb has 4 rows and 4 columns.

The combination of Equations (18), (22), and (23) yields the element stiffness matrix of the beam.

$$
K_{44}=E I_{r} \times T_{b b}
$$

A general matrix formulation of $\mathrm{K}_{44}$ is as follows:

$$
K_{44}=E I_{r} \times\left[\begin{array}{ll}
0 & I
\end{array}\right] \times T^{-1} \times\left[\begin{array}{c}
0^{T} \\
I
\end{array}\right]
$$

In Equation (24b), 0 is a zero matrix with four rows and $2 n+6$ columns, $I$ is the $4 \times 4$ identity matrix. 


\subsubsection{2 $3 \times 3$ element stiffness matrix}

Assuming the presence of a hinge at the right end, the sign convention for bending moments, shear forces, displacements, and slope is illustrated in Figure 11.

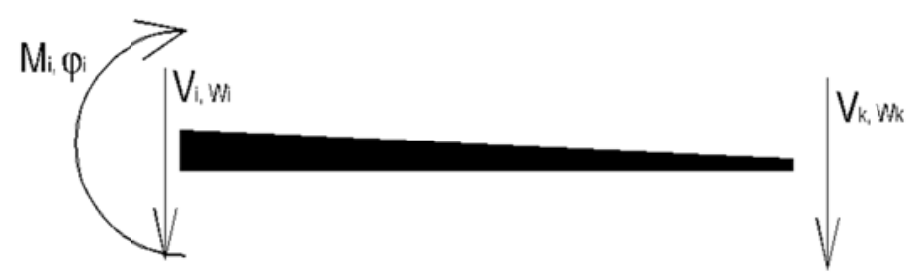

Figure 11 Sign conventions for moments, shear forces, displacements, and slope for stiffness matrix

The $3 \times 3$ element stiffness matrix in local coordinates of the tapered beam is denoted by $\mathrm{K}_{33}$.

The vectors of Equations (17a), (17b), and (18) become

$$
\begin{aligned}
& \overrightarrow{S_{\text {red }}}=\left[V_{i} ; M_{i} ; V_{k}\right]^{T} \\
& \overrightarrow{V_{\text {red }}}=\left[w_{i} ; \varphi_{i} ; w_{k}\right]^{T} \\
& \overrightarrow{S_{\text {red }}}=K_{33} \times \overrightarrow{V_{\text {red }}}
\end{aligned}
$$

The matrix $\mathrm{K}_{33}$ can be formulated with the values of the matrix $\mathrm{K}_{44}$ (see Equations (24a-b)).

$$
K_{44}=\left[\begin{array}{ll}
K_{a a} & K_{a b} \\
K_{b a} & K_{b b}
\end{array}\right]
$$

$\mathrm{K}_{44}$ has 4 rows and 4 columns. The matrix Kaa has 3 rows and 3 columns, the matrix Kab has 3 rows and 1 column, the matrix Kba has 1 row and 3 columns, and the matrix Kbb has 1 row and 1 column (a single value).

The combination of Equation (18) with the presence of a hinge at position $\mathrm{k}\left(\mathrm{M}_{\mathrm{k}}=0\right)$, and Equation (25c) yields the matrix $\mathrm{K}_{33}$ as follows:

$$
K_{33}=K_{a a}-K_{a b} \times \frac{1}{K_{b b}} \times K_{b a}
$$

\subsubsection{First-order element stiffness matrix of a uniform beam}

The beam is divided in $\mathrm{n}$ parts of equal length $\mathrm{h}(\mathrm{l}=\mathrm{nh})$ as shown in Figure 12.
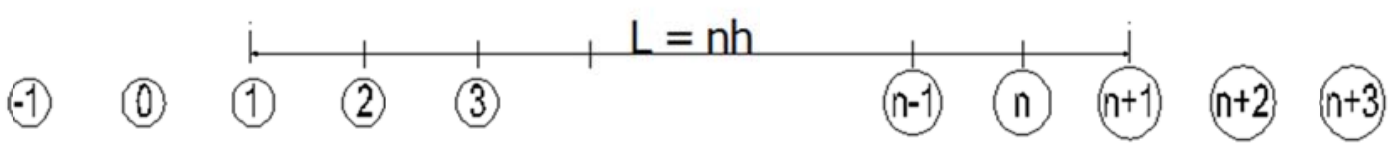

Figure 12 FDM discretization for $4 \mathrm{x} 4$ element stiffness matrix 
Equation (6) with qi $=0$ is applied at any point on the grid (positions $1 ; 2 ; \ldots n+1$ of Figure 12).

The static compatibility boundary conditions (Equations (19a-d)) become

$$
\begin{aligned}
& V_{i}=-V_{1}=-\beta_{k} \times \frac{W_{-1}-2 W_{0}+2 W_{2}-W_{3}}{2 h^{3}} \rightarrow \frac{2 h^{3}}{\beta_{k}} V_{i}+W_{-1}-2 W_{0}+2 W_{2}-W_{3}=0 \\
& M_{i}=M_{1}=\beta_{k} \times \frac{W_{-1}-16 W_{0}+30 W_{1}-16 W_{2}+W_{3}}{12 h^{2}} \\
& \rightarrow \frac{12 h^{2}}{\beta_{k}} M_{i}-W_{-1}+16 W_{0}-30 W_{1}+16 W_{2}-W_{3}=0 \\
& V_{k}=V_{n+1}=\beta_{k} \times \frac{W_{n-1}-2 W_{n}+2 W_{n+2}-W_{n+3}}{2 h^{3}} \\
& \rightarrow \frac{2 h^{3}}{\beta_{k}} V_{k}-W_{n-1}+2 W_{n}-2 W_{n+2}+W_{n+3}=0 \\
& M_{k}=-M_{n+1}=-\beta_{k} \times \frac{W_{n-1}-16 W_{n}+30 W_{n+1}-16 W_{n+2}+W_{n+3}}{12 h^{2}} \\
& \rightarrow \frac{12 h^{2}}{\beta_{k}} M_{k}+W_{n-1}-16 W_{n}+30 W_{n+1}-16 W_{n+2}+W_{n+3}=0
\end{aligned}
$$

The geometric compatibility boundary conditions (Equations (20b,d)) become

$$
\begin{aligned}
& \varphi_{1}=\left.\frac{d w(x)}{d x}\right|_{1}=\frac{w_{-1}-8 w_{0}+8 w_{2}-w_{3}}{12 h}=\varphi_{i} \\
& \rightarrow \frac{W_{-1}-8 W_{0}+8 W_{2}-W_{3}}{12 h}=E I_{r} \times \varphi_{i} \\
& \varphi_{n+1}=\left.\frac{d w(x)}{d x}\right|_{n+1}=\frac{w_{n-1}-8 w_{n}+8 w_{n+2}-w_{n+3}}{12 h}=\varphi_{k} \\
& \rightarrow \frac{W_{n-1}-8 W_{n}+8 W_{n+2}-W_{n+3}}{12 h}=E I_{r} \times \varphi_{k}
\end{aligned}
$$

Equations (20a) and (20c) stay unchanged.

Thus, the number of equations is $n+\mathbf{9}$. The number of unknowns is $n+5+4=\mathbf{n}+\mathbf{9}$, especially $n+5$ unknowns $W$ (at points on the beam and additional points at beam's ends) and 4 efforts at beam’s ends ( $V_{i} ; M_{i} ; V_{k} ; M_{k}$ ).

The vector $\vec{S}_{1}$ becomes,

$$
\vec{S}_{1}=\left[W_{-1} ; W_{0} ; W_{1} \ldots \ldots W_{n+1} ; W_{n+2} ; W_{n+3}\right]^{T}
$$

The use of Equations (22) to (24b) yields the element stiffness matrix of the uniform beam. 


\subsection{Second-order analysis}

The equation of static equilibrium can be expressed as follows:

$$
\frac{d^{2} M(x)}{d x^{2}}+\frac{d}{d x}\left(N(x) \frac{d w(x)}{d x}\right)-k(x) w(x)=-q(x)
$$

The axial force (positive in tension) is denoted by $\mathrm{N}(\mathrm{x})$, and the stiffness of the elastic Winkler foundation by $\mathrm{k}(\mathrm{x})$. Let us also consider an external distributed axial load $\mathrm{n}(\mathrm{x})$ positive along the $+\mathrm{x}$ axis

Combining Equations (30) and (31) yields

$$
n(x)=-\frac{d N(x)}{d x}
$$

$$
\frac{d^{2} M(x)}{d x^{2}}+N(x) \frac{d^{2} w(x)}{d x^{2}}-n(x) \frac{d w(x)}{d x}-k(x) w(x)=-q(x)
$$

\subsubsection{Second-order analysis of a uniform beam within segments}

A beam with constant stiffness in segments was considered. Substituting Equation (2a) into Equation (32) yields

$$
-E I \frac{d^{4} w(x)}{d x^{4}}+N(x) \frac{d^{2} w(x)}{d x^{2}}-n(x) \frac{d w(x)}{d x}-k(x) w(x)=-q(x)
$$

Substituting Equations (4a), (4c), (4d), (5), and (5a) into Equation (33) yields the following governing equation,

$$
\begin{aligned}
& \left(\beta_{i}+\frac{N_{i} h^{2}}{12 E I_{r}}+\frac{n_{i} h^{3}}{12 E I_{r}}\right) W_{i-2}+\left(-4 \beta_{i}-\frac{4 N_{i} h^{2}}{3 E I_{r}}-\frac{2 n_{i} h^{3}}{3 E I_{r}}\right) W_{i-1}+\left(6 \beta_{i}+\frac{5 N_{i} h^{2}}{2 E I_{r}}+\frac{k_{i} h^{4}}{E I_{r}}\right) W_{i} \\
& +\left(-4 \beta_{i}-\frac{4 N_{i} h^{2}}{3 E I_{r}}+\frac{2 n_{i} h^{3}}{3 E I_{r}}\right) W_{i+1}+\left(\beta_{i}+\frac{N_{i} h^{2}}{12 E I_{r}}-\frac{n_{i} h^{3}}{12 E I_{r}}\right) W_{i+2}=q_{i} h^{4}
\end{aligned}
$$

Equation (34) is applied at any point on the grid with spacing h. At point $i$, the external distributed axial load $n_{i}$ is calculated similarly to Equations (8g-i). The transverse force $T(x)$ is related to the shear force $V(x)$ as follows:

$$
T(x)=V(x)+N(x) \frac{d w(x)}{d x}
$$

Substituting Equations (2b), (4b), (4d), (5), and (5a) into (35) yields the FDM formulation of the transverse force

$$
\begin{aligned}
& T_{i}=-\left.E I_{i} \frac{d^{3} w}{d x^{3}}\right|_{i}+\left.N_{i} \frac{d w}{d x}\right|_{i} \\
& \rightarrow 2 h^{3} T_{i}=\left(\beta_{i}+\frac{N h^{2}}{6 E I_{r}}\right) W_{i-2}+\left(-2 \beta_{i}-\frac{4 N h^{2}}{3 E I_{r}}\right) W_{i-1}+\left(2 \beta_{i}+\frac{4 N h^{2}}{3 E I_{r}}\right) W_{i+1}+\left(-\beta_{i}-\frac{N h^{2}}{6 E I_{r}}\right) W_{i+2}
\end{aligned}
$$

The bending moment and the slope are formulated using Equations (7a) and (7c), respectively. 
The analysis at positions of discontinuity is conducted similarly to that of the first-order analysis; however the shear force is replaced by the transverse force.

\subsubsection{Second-order analysis of a tapered beam}

Similarly to the first-order analysis, the M-W and W FDM approximations are considered.

\section{M-W FDM approximation:}

Applying Equations (5), (5a), (14a-b), in Equation (32) yields The FDM formulation of Equation (32) as follows:

$$
\begin{aligned}
& h^{2} M_{i-1}-2 h^{2} M_{i}+h^{2} M_{i+1}+\left(\frac{N_{i} h^{2}}{E I_{r}}+\frac{n_{i} h^{3}}{2 E I_{r}}\right) W_{i-1}-\left(\frac{2 N_{i} h^{2}}{E I_{r}}+\frac{k_{i} h^{4}}{E I_{r}}\right) W_{i} \\
& +\left(\frac{N_{i} h^{2}}{E I_{r}}-\frac{n_{i} h^{3}}{2 E I_{r}}\right) W_{i+1}=-q_{i} h^{4}
\end{aligned}
$$

Equations (37) and (15b) are applied at any point on the grid.

The combination of Equations (35), (15c), and (15d) yields the FDM formulation of the transverse force

$$
2 h^{3} T_{i}=h^{2} M_{i+1}-h^{2} M_{i-1}+\frac{N_{i} h^{2}}{E I_{r}}\left(W_{i+1}-W_{i-1}\right)
$$

The slope is calculated similarly to Equation (15d).

The analysis at positions of discontinuity is conducted similarly to that of the first-order analysis; however the shear force is replaced by the transverse force.

W FDM approximation: Substituting Equation (2a) into (32) yields

$$
-\frac{d^{2}}{d x^{2}}\left[E I(x) \frac{d^{2} w(x)}{d x^{2}}\right]+N(x) \frac{d^{2} w(x)}{d x^{2}}-n(x) \frac{d w(x)}{d x}-k(x) w(x)=-q(x)
$$

Developing Equation (38a) yields

$$
\begin{aligned}
& E I(x) \frac{d^{4} w(x)}{d x^{4}}+2 \frac{d[E I(x)]}{d x} \frac{d^{3} w(x)}{d x^{3}}+\left[\frac{d^{2}[E I(x)]}{d x^{2}}-N(x)\right] \frac{d^{2} w(x)}{d x^{2}} \\
& +n(x) \frac{d w(x)}{d x}+k(x) w(x)=q(x)
\end{aligned}
$$

Applying Equations (15g-j), (4a-d), and (5a) into Equation (15f) yields the following FDM formulation: 


$$
\begin{aligned}
& {\left[\beta_{I i}-\beta_{I i}^{\prime}-\frac{\beta_{I i}^{\prime \prime}}{12}+\frac{N_{i} h_{k}^{2}}{12 E_{r} I_{r}}+\frac{n_{i} h_{k}^{3}}{12 E_{r} I_{r}}\right] W_{i-2}+\left[-4 \beta_{I i}+2 \beta_{I i}^{\prime}+\frac{4 \beta_{I i}^{\prime \prime}}{3}-\frac{4 N_{i} h_{k}^{2}}{3 E_{r} I_{r}}-\frac{2 n_{i} h_{k}^{3}}{3 E_{r} I_{r}}\right] W_{i-1}+} \\
& {\left[6 \beta_{I i}-\frac{5 \beta_{I i}^{\prime \prime}}{2}+\frac{5 N_{i} h_{k}^{2}}{2 E_{r} I_{r}}+\frac{k_{i} h_{k}^{4}}{E_{r} I_{r}}\right] W_{i}+\left[-4 \beta_{I i}-2 \beta_{I i}^{\prime}+\frac{4 \beta_{I i}^{\prime \prime}}{3}-\frac{4 N_{i} h_{k}^{2}}{3 E_{r} I_{r}}+\frac{2 n_{i} h_{k}^{3}}{3 E_{r} I_{r}}\right] W_{i+1}+} \\
& {\left[\beta_{I i}+\beta_{I i}^{\prime}-\frac{\beta_{I i}^{\prime \prime}}{12}+\frac{N_{i} h_{k}^{2}}{12 E_{r} I_{r}}-\frac{n_{i} h_{k}^{3}}{12 E_{r} I_{r}}\right] W_{i-2}=q_{i} h_{k}^{4}}
\end{aligned}
$$

Equation (38c) is applied at any point on the grid.

The bending moment and the rotation of the cross section are calculated using Equations (15l) and (7c), respectively.

The combination of Equations (15m), (35), and (4d) yields the transverse force.

$$
\begin{aligned}
T_{i}= & \beta_{I i}^{\prime} \frac{W_{i-2}-16 W_{i-1}+30 W_{i}-16 W_{i+1}+W_{i+2}}{12 h_{k}^{3}}+\beta_{I i} \frac{W_{i-2}-2 W_{i-1}+2 W_{i+1}-W_{i+2}}{2 h_{k}^{3}}+ \\
& \frac{N_{i} h_{k}^{2}}{E I_{r}} \frac{W_{i-2}-8 W_{i-1}+8 W_{i+1}-W_{i+2}}{12 h_{k}^{3}}
\end{aligned}
$$

\subsubsection{Second-order element stiffness matrix of a tapered beam}

The M-W FDM approximation is used in this section. However, the W FDM approximation could also be considered with appropriate equations (Equations (38c) with $\mathrm{q}_{\mathrm{i}}=0$, (15l), (7c), and (38d)).

The sign conventions for bending moments, transversal forces, displacements, and slopes adopted for use in determining the element stiffness matrix in local coordinates is illustrated in Figure 13.

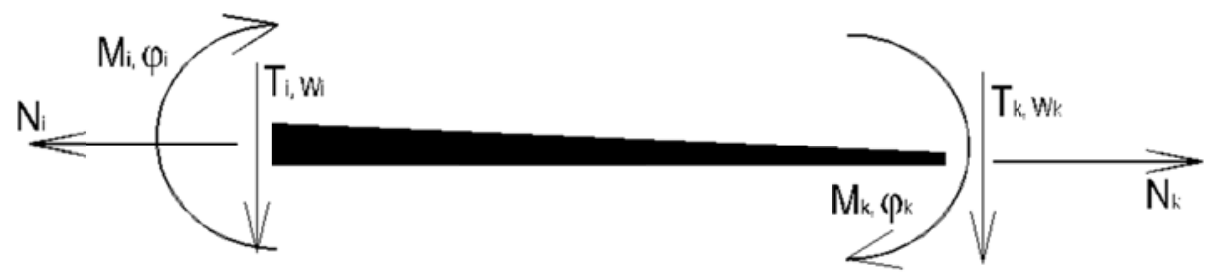

Figure 13 Sign conventions for moments, transversal forces, displacements, and slopes for stiffness matrix

The FDM discretization is the same as Figure 10. Equations (17a) becomes,

$$
\overrightarrow{S_{\text {red }}}=\left[T_{i} ; M_{i} ; T_{k} ; M_{k}\right]^{T}
$$

Equations (37) and (15b) are applied at any point on the grid (the distributed load qi being zero). 
The static compatibility boundary conditions are expressed similarly to Equations (19a-d); however in Equations (19a) and (19c), the shear forces are replaced by the transverse forces (Equation (38)). The analysis continues similar to the first-order element stiffness matrix (Equations (20a-24b)).

\subsection{Vibration analysis of the beam}

\subsubsection{Free vibration analysis}

Our focus here is to determine the eigenfrequencies of the beams. Damping is not considered. A second-order analysis is conducted; the first-order analysis can easily be deduced.

\subsubsection{Free vibration analysis of a uniform beam within segments}

The governing equation is as follows:

$$
E I \frac{\partial^{4} w^{*}(x, t)}{\partial x^{4}}-N(x) \frac{\partial^{2} w^{*}(x, t)}{\partial x^{2}}+n(x) \frac{\partial w^{*}(x, t)}{\partial x}+k(x) w^{*}(x, t)+\rho A \frac{\partial^{2} w^{*}(x, t)}{\partial t^{2}}=0
$$

where $\rho$ is the beam's mass per unit volume, $A$ is the cross-sectional area, $\mathrm{N}(\mathrm{x})$ is the axial force (positive in tension), $\mathrm{n}(\mathrm{x})$ is the external distributed axial load positive along $+\mathrm{x}$ axis, and $\mathrm{k}$ is the stiffness of the elastic Winkler foundation. A harmonic vibration being assumed, $w^{*}(x, t)$ can be expressed as follows:

$$
w^{*}(x, t)=w(x) \times \sin (\omega t+\theta)
$$

Here, $\omega$ is the circular frequency of the beam. Substituting Equation (41) into Equation (40) yields

$$
E I \frac{d^{4} w(x)}{d x^{4}}-N(x) \frac{d^{2} w(x)}{d x^{2}}+n(x) \frac{d w(x)}{d x}+k(x) w(x)-\rho A \omega^{2} w(x)=0
$$

A uniform grid with spacing $h_{k}$ is assumed in the segment $k$.

Substituting Equations (4a), (4c-d), (5), and (5a) into Equation (42) yields the following governing equation:

$$
\begin{aligned}
& \left(\beta_{k}+\frac{N_{i} h_{k}^{2}}{12 E I_{r}}+\frac{n_{i} h_{k}^{3}}{12 E I_{r}}\right) W_{i-2}+\left(-4 \beta_{k}-\frac{4 N_{i} h_{k}^{2}}{3 E I_{r}}-\frac{2 n_{i} h_{k}^{3}}{3 E I_{r}}\right) W_{i-1}+\left(6 \beta_{k}+\frac{5 N_{i} h_{k}^{2}}{2 E I_{r}}+\frac{k_{i} h_{k}^{4}}{E I_{r}}-\frac{\rho A_{k} \omega^{2} h_{k}^{4}}{E I_{r}}\right) W_{i} \\
& +\left(-4 \beta_{k}-\frac{4 N_{i} h_{k}^{2}}{3 E I_{r}}+\frac{2 n_{i} h_{k}^{3}}{3 E I_{r}}\right) W_{i+1}+\left(\beta_{k}+\frac{N_{i} h_{k}^{2}}{12 E I_{r}}-\frac{n_{i} h_{k}^{3}}{12 E I_{r}}\right) W_{i+2}=0
\end{aligned}
$$

Equation (43a) is applied at any point on the grid. The slope, the bending moment, and the transverse force are determined using Equations (7c), (7a), and (36), respectively. 
Let us define a reference length $l_{r}$, a reference cross-sectional area $A_{r}$ and the vibration coefficient $\lambda$ as follows

$$
\begin{aligned}
& h_{k}=\beta_{l k} l_{r} \\
& A_{k}=\beta_{A k} A_{r}
\end{aligned}
$$

$$
\omega=\lambda \times \sqrt{\frac{E I_{r}}{\rho A_{r} l_{r}^{4}}} \rightarrow \frac{\rho A_{k} \omega^{2} h_{k}^{4}}{E I_{r}}=\beta_{A k} \beta_{l k}^{4} \lambda^{2}
$$

A change in grid spacing can be modeled by means of the reference length and the parameters $\beta_{\mathrm{lk}}$.

For the special case of a uniform beam without an axial force or a Winkler foundation, Equation (43a) becomes

$$
W_{i-2}-4 W_{i-1}+\left(6-\beta_{l k}^{4} \lambda^{2}\right) W_{i}-4 W_{i+1}+W_{i+2}=0
$$

\section{Effect of a concentrated mass, or a spring}

We analyzed the dynamic behavior of a beam carrying a concentrated mass or having a spring, as represented in

Figure 14.

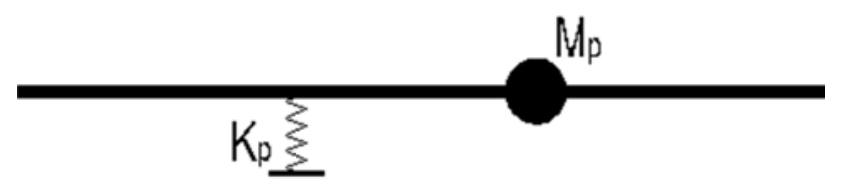

Figure 14 Vibration of beam having a concentrated mass and a spring

The stiffness of the spring is $K_{p}$, and the concentrated mass is $M_{p}$.

$$
\begin{aligned}
& K_{p}=k_{p} \times E I_{r} / l_{r}^{3} \\
& M_{p}=m_{p} \times \rho A_{r} l_{r}
\end{aligned}
$$

The continuity equations for deflection, slope, and bending moment are defined in Equations (10a), (10b), and (11), respectively. Equation (11) is applied with $M^{*}=0$. The reference length of the beam is $l_{r}$ ((Equation (43b)).

Applying Equations (43d) and (44a-b), the balance of vertical forces in case of a concentrated mass or a spring yields

$$
\begin{aligned}
& T_{i l}-T_{i r}-\frac{M_{p} \omega^{2}}{E I_{r}} W_{i l}=0 \rightarrow T_{i l}-T_{i r}-\frac{m_{p}}{l_{r}^{3}} \lambda^{2} W_{i l}=0 \\
& T_{i l}-T_{i r}+\frac{K_{p}}{E I_{r}} W_{i l}=0 \rightarrow T_{i l}-T_{i r}+\frac{k_{p}}{l_{r}^{3}} W_{i l}=0
\end{aligned}
$$

The transverse forces Til and Tir are calculated using Equation (36). 
Effect of a spring-mass system: We analyzed the dynamic behavior of a beam carrying a spring-mass system, as represented in Figure 15. The deflection of the mass is denoted by $\mathrm{W}_{\mathrm{iM}}$.

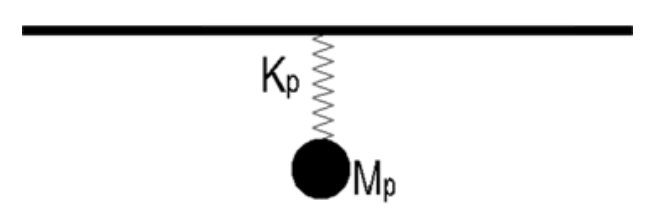

Figure 15 Vibration of a beam carrying a spring-mass system

Applying Equations (43d) and (44a-b), the balance of vertical forces yields

$$
\begin{aligned}
& T_{i l}-T_{i r}-\frac{M_{p} \omega^{2}}{E I_{r}} W_{i M}=0 \rightarrow T_{i l}-T_{i r}-\frac{m_{p}}{l_{r}^{3}} \lambda^{2} W_{i M}=0 \\
& \frac{M_{p} \omega^{2}}{E I_{r}} W_{i M}=\frac{K_{p}}{E I_{r}} \times\left(W_{i M}-W_{i r}\right) \rightarrow m_{p} \lambda^{2} W_{i M}=k_{p}\left(W_{i M}-W_{i r}\right)
\end{aligned}
$$

\subsubsection{Free vibration analysis of tapered beams}

The governing equations are as follows:

$$
\begin{aligned}
& \frac{\partial^{2} M^{*}(x, t)}{\partial x^{2}}+N(x) \frac{\partial^{2} w^{*}(x, t)}{\partial x^{2}}-n(x) \frac{\partial w^{*}(x, t)}{\partial x}-k(x) w^{*}(x, t)-\rho A(x) \frac{\partial^{2} w^{*}(x, t)}{\partial t^{2}}=0 \\
& M^{*}(x, t)=-E I(x) \frac{\partial^{2} w^{*}(x, t)}{\partial x^{2}}
\end{aligned}
$$

A harmonic vibration being assumed, $\mathrm{M}^{*}(\mathrm{x}, \mathrm{t})$ can be expressed similarly to Equation (41). Equations (46a-b) become

$$
\begin{aligned}
& \frac{d^{2} M(x)}{d x^{2}}+N(x) \frac{d^{2} w(x)}{d x^{2}}-n(x) \frac{d w(x)}{d x}-k(x) w(x)+\rho A(x) \omega^{2} w(x)=0 \\
& M(x)=-E I(x) \frac{d^{2} w(x)}{d x^{2}}
\end{aligned}
$$

\section{M-W FDM approximation:}

A uniform grid with spacing $h$ is assumed. The grid at the beam's ends and at positions of discontinuity is the same as represented in Figure 8a,b. Substituting Equations (5a), (14a-b), and (43c) into Equations (47b) and (47a) yields Equation (15b) and the following equation:

$$
\begin{aligned}
& h^{2} M_{i-1}-2 h^{2} M_{i}+h^{2} M_{i+1}+\left(\frac{N_{i} h^{2}}{E I_{r}}+\frac{n_{i} h^{3}}{2 E I_{r}}\right) W_{i-1}-\left(\frac{2 N_{i} h^{2}}{E I_{r}}+\frac{k_{i} h^{4}}{E I_{r}}-\beta_{A i} \frac{\rho A_{r} \omega^{2} h^{4}}{E I_{r}}\right) W_{i} \\
& +\left(\frac{N_{i} h^{2}}{E I_{r}}-\frac{n_{i} h^{3}}{2 E I_{r}}\right) W_{i+1}=0
\end{aligned}
$$


The application of Equations (43b, d) yields

$$
\omega=\lambda \times \sqrt{\frac{E I_{r}}{\rho A_{r} l_{r}^{4}}} \rightarrow \frac{\rho A_{r} \omega^{2} h^{4}}{E I_{r}}=\beta_{l}^{4} \lambda^{2}
$$

For the special case of a tapered beam without an axial force or a Winkler foundation, Equation (48a) becomes

$$
h^{2} M_{i-1}-2 h^{2} M_{i}+h^{2} M_{i+1}+\beta_{A i} \beta_{l}^{4} \lambda^{2} W_{i}=0
$$

Equations (15b) and (48a) are applied at any point on the grid. The slope and the transverse force are determined using Equations (15d) and (38), respectively.

\section{Effect of a concentrated mass, a spring, or a spring-mass system}

The analysis is conducted similarly to the section above (Equations (44a) to (45d)); the transverse forces Til and Tir in Equations (45a-c) are calculated using Equation (38).

\section{W FDM approximation:}

Substituting Equation (47b) into (47a) and developments yields

$$
\begin{aligned}
& E I(x) \frac{d^{4} w(x)}{d x^{4}}+2 \frac{d[E I(x)]}{d x} \frac{d^{3} w(x)}{d x^{3}}+\left[\frac{d^{2}[E I(x)]}{d x^{2}}-N(x)\right] \frac{d^{2} w(x)}{d x^{2}} \\
& +n(x) \frac{d w(x)}{d x}+\left[k(x)-\omega^{2} \rho(x) A(x)\right] w(x)=0
\end{aligned}
$$

Applying Equations (15g-j), (4a-d), (5a), (43c-d) into Equation (48d) yields

$$
\begin{aligned}
& {\left[\beta_{I i}-\beta_{I i}^{\prime}-\frac{\beta_{I i}^{\prime \prime}}{12}+\frac{N_{i} h_{k}^{2}}{12 E_{r} I_{r}}+\frac{n_{i} h_{k}^{3}}{12 E_{r} I_{r}}\right] W_{i-2}+\left[-4 \beta_{I i}+2 \beta_{I i}^{\prime}+\frac{4 \beta_{I i}^{\prime \prime}}{3}-\frac{4 N_{i} h_{k}^{2}}{3 E_{r} I_{r}}-\frac{2 n_{i} h_{k}^{3}}{3 E_{r} I_{r}}\right] W_{i-1}+} \\
& {\left[6 \beta_{I i}-\frac{5 \beta_{I i}^{\prime \prime}}{2}+\frac{5 N_{i} h_{k}^{2}}{2 E_{r} I_{r}}+\frac{k_{i} h_{k}^{4}}{E_{r} I_{r}}-\beta_{I k}^{4} \beta_{A i} \lambda^{2}\right] W_{i}+\left[-4 \beta_{I i}-2 \beta_{I i}^{\prime}+\frac{4 \beta_{I i}^{\prime \prime}}{3}-\frac{4 N_{i} h_{k}^{2}}{3 E_{r} I_{r}}+\frac{2 n_{i} h_{k}^{3}}{3 E_{r} I_{r}}\right] W_{i+1}+} \\
& {\left[\beta_{I i}+\beta_{I i}^{\prime}-\frac{\beta_{I i}^{\prime \prime}}{12}+\frac{N_{i} h_{k}^{2}}{12 E_{r} I_{r}}-\frac{n_{i} h_{k}^{3}}{12 E_{r} I_{r}}\right] W_{i-2}=0}
\end{aligned}
$$


The bending moment, the transverse force, and the rotation of the cross section are calculated using Equations (15l), (38d), and (7c), respectively.

For the special case of a tapered beam without an axial force or a Winkler foundation, Equation (48e) becomes

$$
\begin{aligned}
& {\left[\beta_{I i}-\beta_{I i}^{\prime}-\frac{\beta_{I i}^{\prime \prime}}{12}\right] W_{i-2}+\left[-4 \beta_{I i}+2 \beta_{I i}^{\prime}+\frac{4 \beta_{I i}^{\prime \prime}}{3}\right] W_{i-1}+} \\
& {\left[6 \beta_{I i}-\frac{5 \beta_{I i}^{\prime \prime}}{2}-\beta_{I k}^{4} \beta_{A i} \lambda^{2}\right] W_{i}+\left[-4 \beta_{I i}-2 \beta_{I i}^{\prime}+\frac{4 \beta_{I i}^{\prime \prime}}{3}\right] W_{i+1}+\left[\beta_{I i}+\beta_{I i}^{\prime}-\frac{\beta_{I i}^{\prime \prime}}{12}\right] W_{i-2}=0}
\end{aligned}
$$

\subsubsection{Direct time integration method}

The direct time integration method developed here describes the dynamic response of the beam as multi-degree-offreedom system. The damping (viscosity $\eta$ ) and an external loading $\mathrm{p}(\mathrm{x}, \mathrm{t})$ are considered.

Uniform beam : $\quad$ The governing equation is applied at any point on the beam as follows:

$$
\begin{aligned}
& E I \frac{\partial^{4} w^{*}(x, t)}{\partial x^{4}}-N(x) \frac{\partial^{2} w^{*}(x, t)}{\partial x^{2}}+n(x) \frac{\partial w^{*}(x, t)}{\partial x}+k(x) w^{*}(x, t)= \\
& p(x, t)-\rho A \frac{\partial^{2} w^{*}(x, t)}{\partial t^{2}}-\eta \frac{\partial w^{*}(x, t)}{\partial t}
\end{aligned}
$$

The derivatives with respect to $\mathrm{x}$ are formulated using Equations (4a), (4c), and (4d); those with respect to $\mathrm{t}$ (the time increment is $\Delta \mathrm{t}$ ) are formulated considering a three-point stencil with Equations (50a-c),

$$
\left.\frac{\partial w^{*}(x, t)}{\partial t}\right|_{i, t}=\left.\frac{-w_{i, t-\Delta t}^{*}+w_{i, t+\Delta t}^{*}}{2 \Delta t} \quad \frac{\partial^{2} w^{*}(x, t)}{\partial t^{2}}\right|_{i, t}=\frac{w_{i, t-\Delta t}^{*}-2 w_{i, t}^{*}+w_{i, t+\Delta t}^{*}}{\Delta t^{2}}
$$

At the initial time $\mathbf{t}=\mathbf{0}$, a three-point forward difference approximation (Equation (8b)) is applied

$$
\left.\frac{\partial^{2} w^{*}}{\partial t^{2}}\right|_{i, 0}=\left.\frac{w_{i, 0}^{*}-2 w_{i, \Delta t}^{*}+w_{i, 2 \Delta t}^{*}}{\Delta t^{2}} \quad \frac{\partial w^{*}}{\partial t}\right|_{i, 0}=\frac{-3 w_{i, 0}^{*}+4 w_{i, \Delta t}^{*}-w_{i, 2 \Delta t}^{*}}{2 \Delta t}
$$

At the final time $\mathbf{t}=\mathbf{T}$, a three-point backward difference approximation (Equation (8d)) is applied

$$
\left.\frac{\partial^{2} w^{*}}{\partial t^{2}}\right|_{i, T}=\left.\frac{w_{i, T-2 \Delta t}^{*}-2 w_{i, T-\Delta t}^{*}+w_{i, T}^{*}}{\Delta t^{2}} \quad \frac{\partial w^{*}}{\partial t}\right|_{i, T}=\frac{w_{i, T-2 \Delta t}^{*}-4 w_{i, T-\Delta t}^{*}+3 w_{i, T}^{*}}{2 \Delta t}
$$

The governing equation (Equation (49)) can be formulated with FDM for $\mathrm{x}=\mathrm{i}$ at time $\mathrm{t}$. The FDM formulation of this equation is applied at any point of the beam at any time t using a seven-point stencil. Additional points are introduced 
to satisfy the boundary and continuity conditions. The boundary conditions are satisfied using a five-point stencil. Thus, the beam deflection $\mathrm{w}^{*}(\mathrm{x}, \mathrm{t})$ can be determined with the Cartesian model represented in Figure 16. The bending moments $\mathrm{M}^{*}(\mathrm{x}, \mathrm{t})$ and the shear force $\mathrm{V}^{*}(\mathrm{x}, \mathrm{t})$ are calculated using Equations (7a,b).

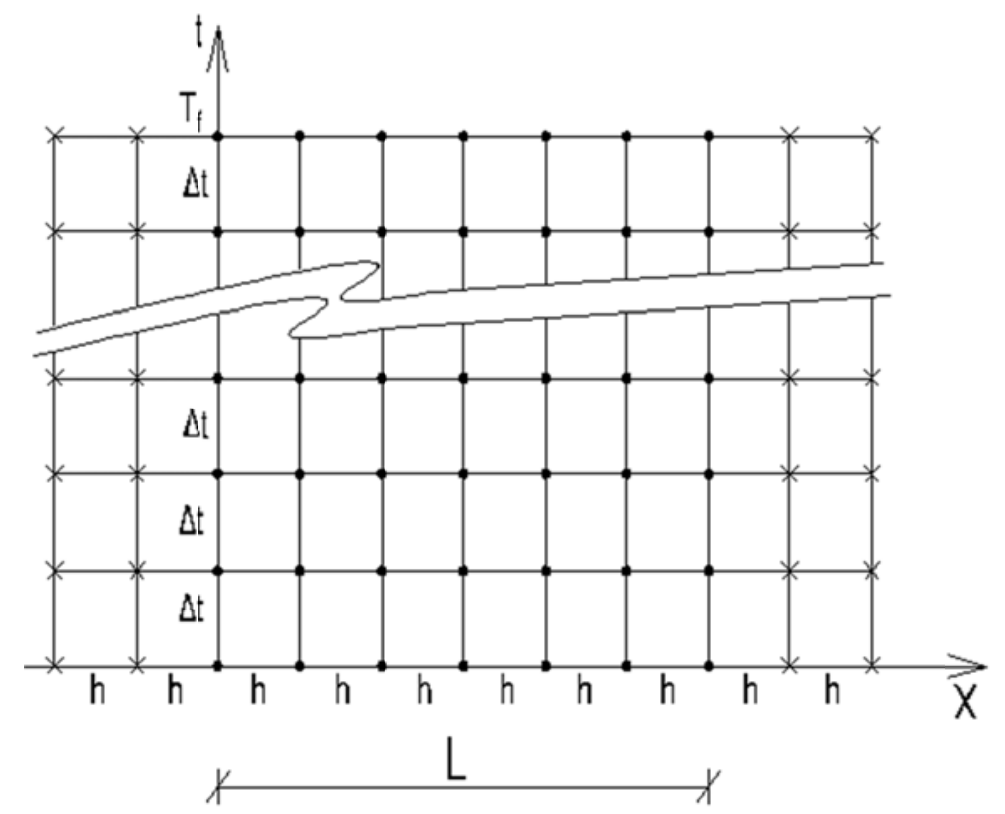

Figure 16 Model for the calculation of time-dependent vibration of a uniform beam

Tapered beam: a similar analysis is conducted. The M-W FDM approximation is applied; however, the W FDM approximation could also be considered with appropriate equations. Thus, Equation (49) becomes

$$
\begin{aligned}
& \frac{\partial^{2} M^{*}(x, t)}{\partial x^{2}}+N(x) \frac{\partial^{2} w^{*}(x, t)}{\partial x^{2}}-n(x) \frac{\partial w^{*}(x, t)}{\partial x}-k(x) w^{*}(x, t)= \\
& -p(x, t)+\rho A(x) \frac{\partial^{2} w^{*}(x, t)}{\partial t^{2}}+\eta \frac{\partial w^{*}(x, t)}{\partial t}
\end{aligned}
$$

The derivatives with respect to $\mathrm{x}$ are formulated using Equations (14a-b); those with respect to $\mathrm{t}$ are formulated with Equations (50a-c).

The FDM formulation of Equations (51) and (46b) are applied at any point on the beam and at any time $\mathrm{t}$ using a fivepoint stencil and a three-point stencil, respectively. Additional points are introduced to satisfy the boundary and continuity conditions. The boundary conditions are satisfied using a three-point stencil. Thus, the bending moment $\mathrm{M}^{*}(\mathrm{x}, \mathrm{t})$ and beam deflection $\mathrm{w}^{*}(\mathrm{x}, \mathrm{t})$ can be determined with the Cartesian model represented in Figure 17. The transverse force $\mathrm{T}^{*}(\mathrm{x}, \mathrm{t})$ is calculated using Equation (38). 


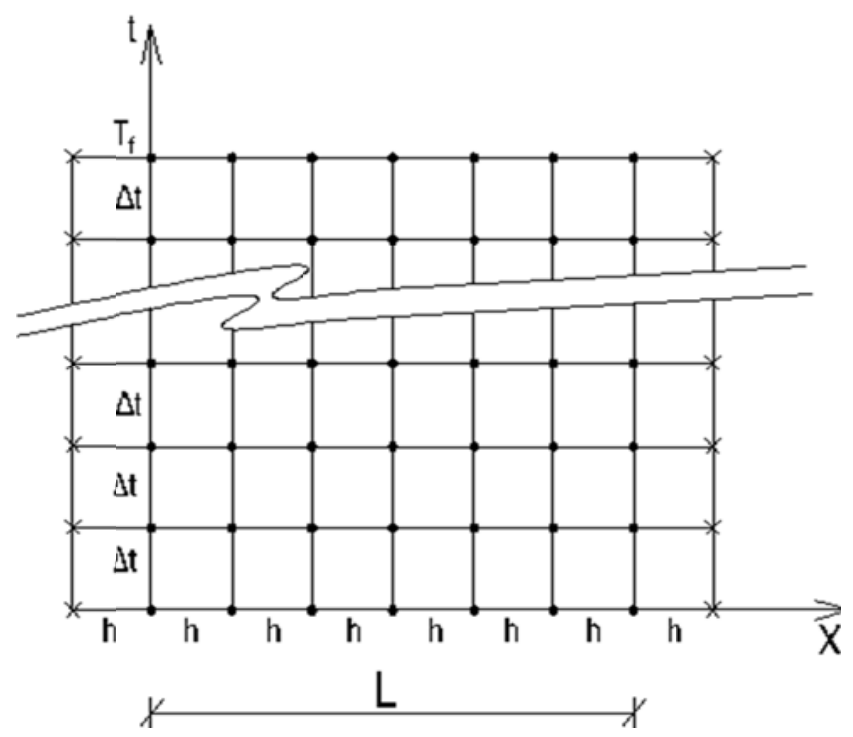

Figure 17 Model for the calculation of time-dependent vibration of a tapered beam

With the direct time integration method developed here, the assumptions previously made can be verified, namely the separation of variables and the harmonic vibration (Equation (41)).

\subsection{Extrapolation to approximate the exact result}

The analysis with the FDM is an approximation. Generally, the accuracy of the results increases by increasing the number of grid points; therefore when the number of points is infinitely high, the results converge towards the exact results. It is assumed that the relationship between the results $\mathrm{R}$ and the number $\mathrm{N}$ of grid points on the beam follows a hyperbolic curve with the constants A, B, and C, as follows:

$$
R=\frac{A N+B}{N+C}
$$

Three couples of values $\left(\mathrm{N}_{\mathrm{i}} ; \mathrm{R}_{\mathrm{i}}\right)$ are then necessary to determine $\mathrm{A}, \mathrm{B}$, and C. Solving the following equation system yields A, B, and C.

$$
\begin{aligned}
& A N_{1}+B-R_{1} C=R_{1} N_{1} \\
& A N_{2}+B-R_{2} C=R_{2} N_{2} \\
& A N_{3}+B-R_{3} C=R_{3} N_{3}
\end{aligned}
$$

The exact result Re is approximated when the number of grid points $\mathrm{N} \rightarrow \propto$ :

$$
\mathrm{R}_{\mathrm{e}}=\lim _{N \rightarrow \infty} \frac{A N+B}{N+C}=A
$$

Higher-order interpolations can also be considered as follows: 


$$
R=\frac{A N^{2}+B N+C}{N^{2}+D N+E}
$$

In this case five couples of values $\left(\mathrm{N}_{\mathrm{i}} ; \mathrm{R}_{\mathrm{i}}\right)$ are necessary to determine $\mathrm{A}, \mathrm{B}, \mathrm{C}, \mathrm{D}$, and $\mathbf{E}$.

\section{$3 \quad$ Results and discussions}

\subsection{First-order analysis}

\subsubsection{Beam subjected to a uniformly distributed load}

We analyzed a uniform fixed-pinned beam subjected to a uniformly distributed load, as shown in Figure 18.

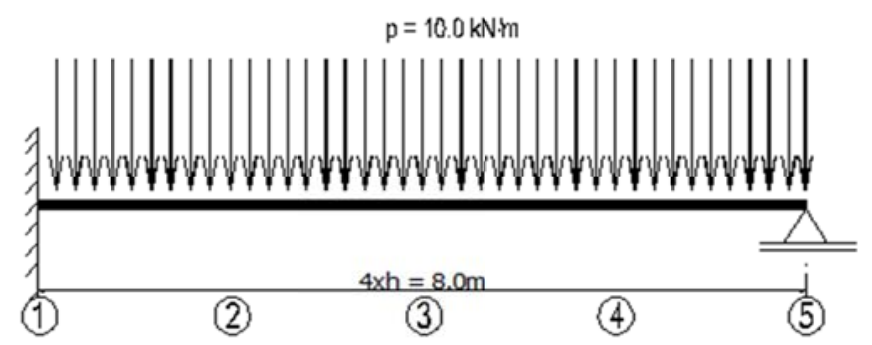

(6)

Figure 18 Uniform fixed-pinned beam subjected to a uniformly distributed load

The governing equation (Equation (6)) was applied at grid points 1, 2, 3, 4, and 5. The bioundary conditions were satisfied using Equations (7a) and (7c). The analysis and results are detailed in the supplementary material “fixed-pinned beam subjected to a uniformly distributed load.” Table 1 lists the results obtained with the classical beam theory (CBT) and those obtained in the present study. Furthermore, the results are presented for a three-point stencil (TPS) considered for the slope (Equation (14b)) and bending moment (Equation (14a)) when satisfying the boundary conditions. Finally the results for a number of grid points $n=4,3$, and 2 are presented.

Table 1 Bending moments $(\mathrm{kNm})$ in the beam for various number of grid points: classical beam theory (CBT), present study, present study (three-point stencil (TPS))

\begin{tabular}{lcccccc}
\hline \multicolumn{3}{c}{ Five-point grid } & & \multicolumn{3}{c}{ Four-point grid } \\
& \multicolumn{3}{c}{$4 \times 2.0 \mathrm{~m}$} & & $3 \times 2.67 \mathrm{~m}$ \\
\hline Position & CBT & Present study & Present study & Position & CBT & Present study \\
$\mathbf{X}(\mathbf{m})$ & (exact results) & & (TPS) & $\mathbf{X}(\mathbf{m})$ & (exact results) & \\
\hline 0.0 & -80.00 & -80.00 & -72.73 & 0.00 & -80.00 & -80.00 \\
2.0 & 0.00 & 0.00 & 5.45 & 2.67 & 17.78 & 17.78 \\
4.0 & 40.00 & 40.00 & 43.64 & 5.33 & 44.44 & 44.44 \\
6.0 & 40.00 & 40.00 & 41.82 & 8.00 & 0.00 & 0.00 \\
8.0 & 0.00 & 0.00 & 0.00 & & & \\
\hline
\end{tabular}


EULER-BERNOULLI BEAM THEORY USING THE FINITE DIFFERENCE METHOD

\begin{tabular}{cccccc}
\hline \multicolumn{3}{c}{ Three-point grid } & & Two-point grid & \\
& $2 \times 4.0 \mathrm{~m}$ & & & $1 \times 8.0 \mathrm{~m}$ & \\
\hline Position & CBT & Present study & Position & CBT & Present study \\
$\mathbf{X}(\mathbf{m})$ & $($ exact results) & & $\mathbf{X}(\mathbf{m})$ & $($ exact results $)$ & \\
\hline 0.00 & -80.00 & -80.00 & 0.00 & -80.00 & -80.00 \\
4.00 & 40.00 & 40.00 & 8.00 & 0.00 & 0.00 \\
8.00 & 0.00 & 0.00 & & & \\
\hline
\end{tabular}

The results of the present study are exact for the beam subjected to a uniformly distributed load regardless of the discretization, since the exact solution for the deflection curve is here a fourth-order polynomial which corresponds to the FDM polynomial hypothesis. It is noted that the use of a three-point stencil for the bending moment and slope yields less accurate results since here the finite difference approximations are derived based on a second-order polynomial hypothesis for the deflection curve, while the deflection curve is a fourth-order polynomial.

\subsubsection{Beam subjected to a concentrated load}

A uniform fixed-pinned beam subjected to a concentrated load, as represented in Figure 19, was analyzed.

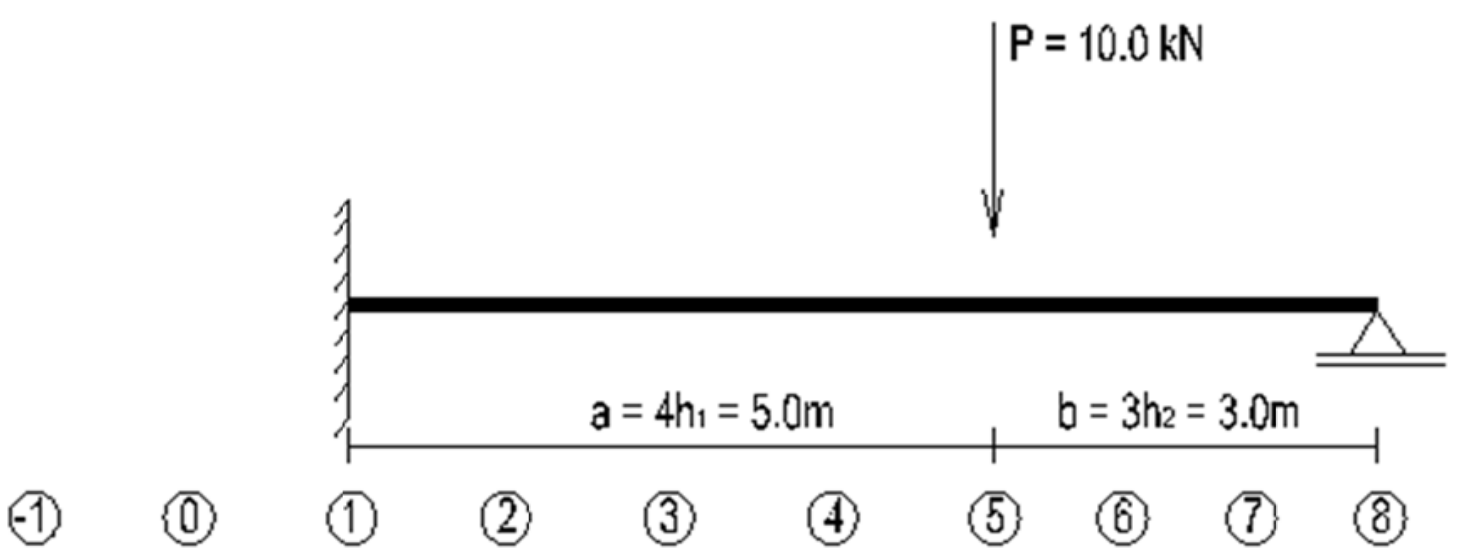

Figure 19 Uniform fixed-pinned beam subjected to a concentrated load

The model showing the grid points at the position of the load, as shown in Figure 4a,b, was considered.

The governing equation (Equation (6)) was applied at grid points 1, 2, 3, 4, 5l, 5r, 6, 7, and 8. The boundary and continuity conditions were satisfied using Equations (7a) and (7c), and Equations (10a) to (12), respectively. Details of the analysis and results are presented in the supplementary material "fixed-pinned beam subjected to a concentrated load.” Table 2 displays the results obtained with the classical beam theory (CBT) and those obtained with the present study (FDM). In addition, the results for a three-point discretization of the beam are also indicated. 
Table 2 Bending moments $(\mathrm{kNm})$ in the beam: CBT, FDM

Eight-point grid Three-point grid

\begin{tabular}{lcccc} 
& \multicolumn{2}{c}{$5 \times 1.25 \mathrm{~m}+3 \times 1.0 \mathrm{~m}+3.0 \mathrm{~m}$} & Position & FDM \\
\hline $\mathbf{X}(\mathbf{m})$ & CBT & FDM & $\mathbf{X}(\mathbf{m})$ & \\
\hline 0.00 & -12.89 & & 0.00 & \\
1.25 & -6.19 & -12.89 & & \\
2.50 & 0.51 & -6.19 & & 13.92 \\
3.75 & 7.21 & 0.51 & & \\
5.00 & 13.92 & 7.21 & 5.00 & \\
6.00 & 9.28 & 13.92 & & \\
7.00 & 4.64 & 9.28 & & \\
8.00 & 0.00 & 4.64 & & \\
\hline
\end{tabular}

The results of the present study are exact for the beam subjected to a concentrated load regardless of the discretization, since the exact solution for the deflection curve is here a third-order polynomial which is exactly described with the fourth-order polynomial FDM approximation.

\subsubsection{Beam subjected to a linearly distributed load}

The analysis of a uniform fixed-pinned beam subjected to a linearly distributed load, as shown in Figure 20, was conducted.

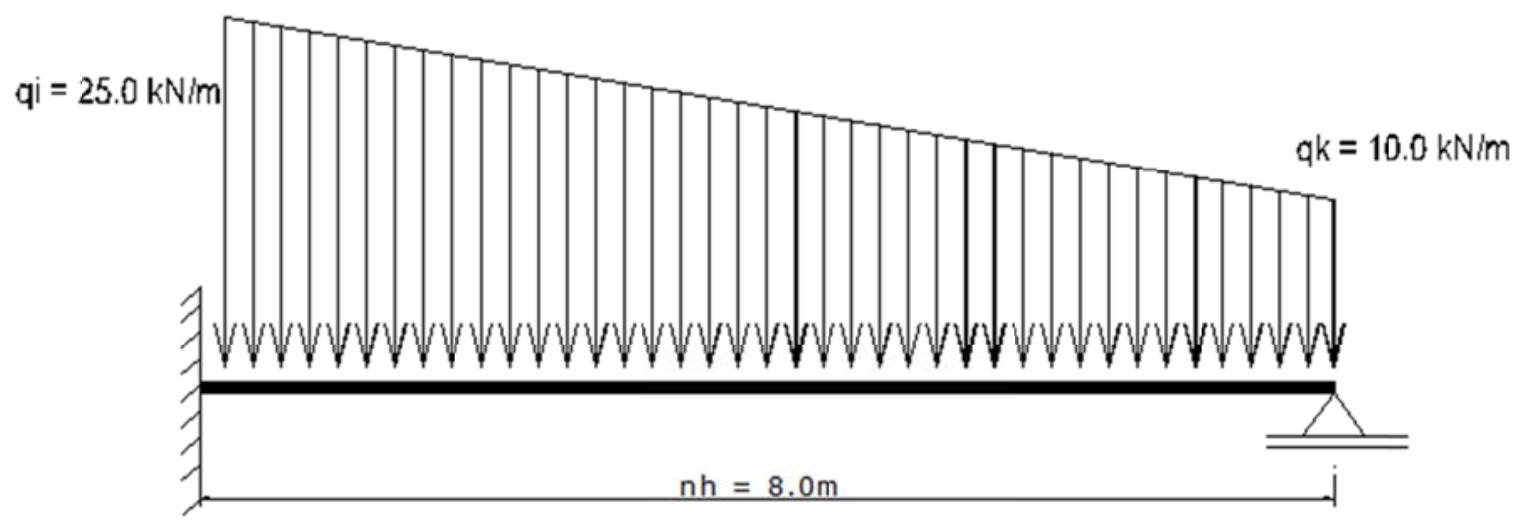

Figure 20 Uniform fixed-pinned beam subjected to a linearly distributed load

The beam was calculated using a five-point grid and a six-point grid. 
The analysis and results are detailed in the supplementary material "fixed-pinned beam subjected to a linearly distributed load.” Table 3 shows the results obtained with the classical beam theory (the exact results) and those obtained in the present study (FDM).

Table 3 Bending moments $(\mathrm{kNm})$ in the beam: CBT, FDM

\begin{tabular}{|c|c|c|c|c|c|c|c|}
\hline Position & CBT & FDM & Difference & Position & CBT & FDM & Difference \\
\hline$X(m)$ & (exact results) & Five-point grid & $\%$ & $X(m)$ & (exact results) & Six-point grid & $\%$ \\
\hline 0.00 & -144.00 & -144.38 & 0.26 & 0.00 & -144.00 & -144.15 & 0.10 \\
\hline 2.00 & 4.50 & 4.22 & -6.22 & 1.60 & -17.92 & -18.04 & 0.67 \\
\hline 4.00 & 68.00 & 67.81 & -0.28 & 3.20 & 51.84 & 51.75 & -0.17 \\
\hline 6.00 & 61.50 & 61.41 & -0.15 & 4.80 & 72.96 & 72.90 & -0.08 \\
\hline \multirow[t]{2}{*}{8.00} & 0.00 & 0.00 & & 6.40 & 53.12 & 53.09 & -0.06 \\
\hline & & & & 8.00 & 0.00 & 0.00 & \\
\hline
\end{tabular}

As Table 3 shows, the results of the present study have a high accuracy. It is noted that the exact results cannot be get since the exact solution of $\mathrm{w}(\mathrm{x})$ for a linearly distributed loading is a fifth-order polynomial while the FDM approximation is a fourth-order polynomial. However the accuracy increases with increasing number of grid points. The exact results could be obtained by means of a polynomial refinement, namely a fifth-order polynomial hypothesis for the deflection curve, whereby non-centered finite difference approximations at beam's ends would be used; further research will be needed for this purpose.

\subsubsection{Tapered pinned-fixed beam subjected to a uniformly distributed load}

We analyzed a tapered pinned-fixed beam subjected to a uniformly distributed load, as shown in Figure 21.

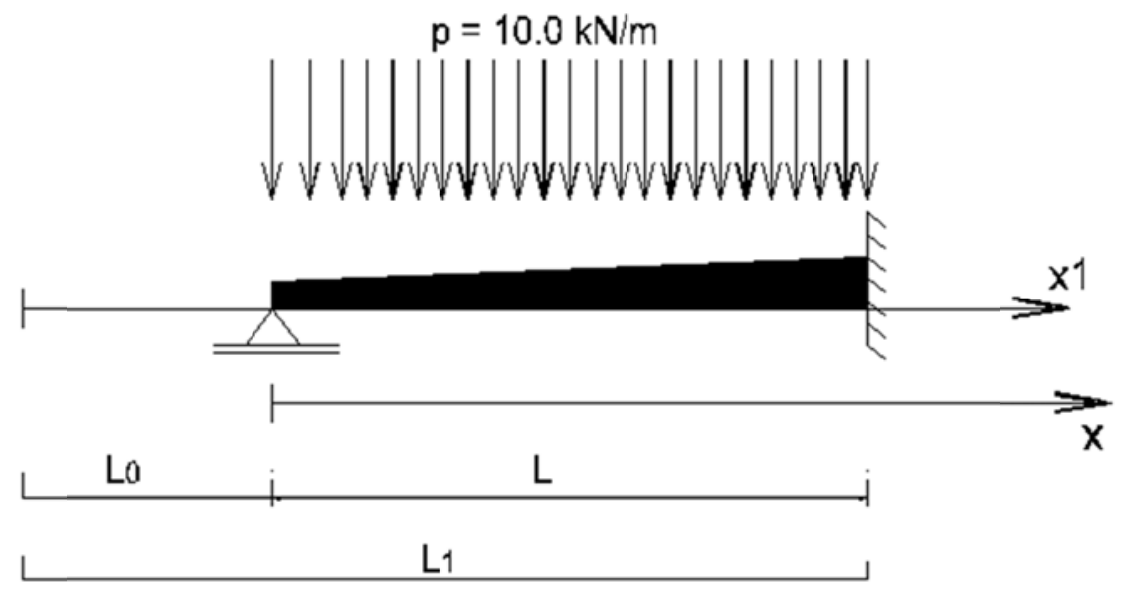

Figure 21 Tapered pinned-fixed beam subjected to a uniformly distributed load 
At a position $\mathrm{x}_{1}$ of the beam the second moment of area $\mathrm{I}(\mathrm{x} 1)$ is defined as follows,

$$
I\left(x_{1}\right)=I_{1}\left(x_{1} / L_{1}\right)^{4}
$$

$\mathrm{I}_{1}$ is the second moment of area at $\mathrm{x}_{1}=\mathrm{L}_{1}$.

Given $\mathrm{L}=8.0 \mathrm{~m}$ and $\mathrm{L}_{0}=2.0 \mathrm{~m}$

First, the beam was calculated with the force method of the classical beam theory (exact results). Then, the calculation was conducted using the M-W FDM and W FDM approximations with $n=9,13$, and 17 grid points. Due to the low rate of convergence of the W FDM approximation, the calculation was extended to $n=25,33,49$, and 65 grid points. Details of the analysis and results are presented in Appendix A and in the supplementary material "tapered pinned-fixed beam subjected to a uniformly distributed load.” Table 4 lists the results obtained with the classical beam theory and those obtained in the present study (M-W FDM and W FDM approximations).

Table 4 Bending moments ( $\mathrm{kNm})$ in the tapered beam: CBT, M-W FDM, and W FDM approximations

\begin{tabular}{|c|c|c|c|c|c|c|c|}
\hline \multirow{2}{*}{$\begin{array}{l}\text { Position } \\
\mathrm{X}(\mathrm{m})\end{array}$} & \multirow{2}{*}{$\begin{array}{c}\text { CBT } \\
\text { (exact results) }\end{array}$} & \multicolumn{2}{|c|}{ Nine-point grid } & \multicolumn{2}{|c|}{ Thirteen-point grid } & \multicolumn{2}{|c|}{ Seventeen-point grid } \\
\hline & & M-W FDM & W FDM & M-W FDM & W FDM & M-W FDM & W FDM \\
\hline 0.00 & 0.00 & 0.00 & 0.00 & 0.00 & 0.00 & 0.00 & 0.00 \\
\hline 2.00 & 17.36 & 17.70 & 1.37 & 17.45 & 14.40 & 17.39 & 16.04 \\
\hline 4.00 & -5.29 & -4.61 & -10.89 & -5.11 & -8.65 & -5.22 & -9.19 \\
\hline 6.00 & -67.93 & -66.91 & -63.02 & -67.66 & -67.66 & -67.83 & -71.91 \\
\hline 8.00 & -170.58 & -169.22 & -152.46 & -170.22 & -165.15 & -170.44 & -173.71 \\
\hline \multirow{2}{*}{$\begin{array}{c}\text { Position } \\
\mathrm{X}(\mathrm{m})\end{array}$} & CBT & \multicolumn{2}{|r|}{ 25-point grid } & 33-point grid & \multicolumn{2}{|c|}{ 49-point grid } & 65-point grid \\
\hline & (exact results) & & W FDM & W FDM & \multicolumn{2}{|c|}{ W FDM } & W FDM \\
\hline 0.00 & 0.00 & & 0.00 & 0.00 & \multicolumn{2}{|c|}{0.00} & 0.00 \\
\hline 2.00 & 17.36 & & 16.75 & 17.03 & \multicolumn{2}{|c|}{17.22} & 17.28 \\
\hline 4.00 & -5.29 & & -5.19 & -5.38 & \multicolumn{2}{|c|}{-5.35} & -5.33 \\
\hline 6.00 & -67.93 & & -66.03 & -67.15 & \multicolumn{2}{|c|}{-67.63} & -67.77 \\
\hline 8.00 & -170.58 & & -166.43 & -168.68 & $-16 s$ & & 0.15 \\
\hline
\end{tabular}

The results of this study are in good agreement with the exact results. The M-W FDM approximation converges faster towards the exact results than the W FDM approximation. Interestingly, it is noted that the negative bending moments with W FDM converge towards the exact results in a non-monotonous manner; this phenomenon should be investigated in further research. 


\subsection{Second-order analysis}

\subsubsection{Beam subjected to a uniformly distributed load and a compressive force}

A uniform fixed-free beam subjected to a uniformly distributed load and a compressive force, as shown in Figure 22, was analyzed.

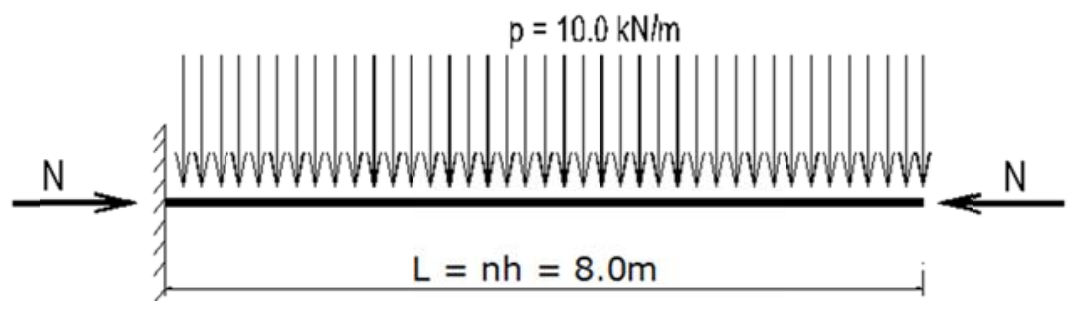

Figure 22 Fixed-free beam subjected to a uniformly distributed load and a compressive force

$$
\frac{N l^{2}}{E I}=-1.50
$$

The governing equation (Equation (6)) was applied at grid points. The analysis was carried out with $n=9,13$, and 17 grid points; then the results were extrapolated to obtain those for infinite grid points (Equation (54)). Details of the analysis and results are presented in the supplementary material "fixed-free beam subjected to a uniformly distributed load and compressive force.” Table 5 displays the results obtained with the classical beam theory (CBT) and those obtained in the present study (FDM).

Table 5 Bending moments $(\mathrm{kNm})$ in the fixed-free beam: CBT, FDM

\begin{tabular}{lccccc}
\hline Position & CBT & FDM & FDM & FDM & FDM \\
X(m) & (exact results) & Nine-point grid & Thirteen-point grid & Seventeen-point grid & N $=\infty$ \\
\hline 0.00 & -618.05 & -625.45 & -621.31 & -619.88 & -616.93 \\
2.00 & -451.63 & -457.83 & -454.36 & -453.16 & -450.70 \\
4.00 & -282.90 & -287.31 & -284.84 & -283.919 & -282.23 \\
6.00 & -127.54 & -129.79 & -128.53 & -128.09 & -127.20 \\
8.00 & 0.00 & 0.00 & 0.00 & 0.00 & 0.00
\end{tabular}

The results of the present study have a high accuracy. The extrapolation towards the exact results $(\mathrm{n}=\infty)$ delivers good results.

\subsubsection{Buckling load of a fixed-pinned beam}

We determined the buckling load of a fixed-pinned beam, as shown in Figure 23.

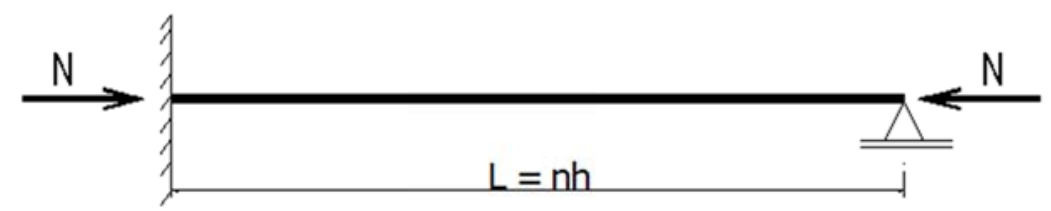

Figure 23 Buckling load of a fixed-pinned beam 
The FDM analysis was conducted using $n=9,13$, and 17 grid points. The results were then extrapolated to obtain those for infinite grid points. The analysis and results are detailed in the supplementary material "stability of a fixed-pinned beam.” The buckling load $\mathrm{N}_{\mathrm{cr}}$ is defined as follows:

$$
N_{c r}=-\pi^{2} E I /(\beta l)^{2}
$$

Values of the buckling factor $\beta$ are listed in Table 6.

Table 6 Buckling factors of the beam: CBT, present study

\begin{tabular}{ccccc}
\hline $\begin{array}{c}\text { CBT } \\
\text { (exact results) }\end{array}$ & FDM & FDM & FDM & FDM \\
\hline 0.699 & Nine-point grid & Thirteen-point grid & Seventeen-point grid & $\mathrm{n}=\infty$ \\
\hline
\end{tabular}

The results of the present study have a high accuracy.

\subsubsection{Buckling load of a tapered beam}

The buckling loads of tapered beams with various support conditions, as shown in Figure 21, were determined.

The analysis was carried out with $n=9,13$, and 17 grid points; then the results were extrapolated to obtain those for infinite grid points. The analysis and results are detailed in the supplementary material "stability of a tapered beam." The buckling load $\mathrm{N}_{\mathrm{cr}}$ is defined as follows:

$$
N_{c r}=-\pi^{2} E I_{1} /(\beta l)^{2}
$$

The buckling factors $\beta$ are listed in Table 7 for $\xi_{0}=\mathrm{L}_{0} / \mathrm{L}_{1}=0.25$

Table 7 Buckling factors $\beta$ of tapered beams with $\xi_{0}=\mathrm{L}_{0} / \mathrm{L}_{1}=0.25$

\begin{tabular}{ccccc}
\hline & FDM & FDM & FDM & FDM \\
& Nine-point grid & Thirteen-point grid & Seventeen-point grid & $\mathrm{n}=\infty$ \\
\hline Pinned-pinned & 4.0255 & 4.0249 & 4.0167 & 4.0263 \\
Pinned-fixed & 2.8403 & 2.8411 & 2.8268 & 2.8396 \\
Free-fixed & 5.1245 & 5.1284 & 5.1310 & 5.1452 \\
Fixed-fixed & 2.4584 & 2.2153 & 2.1017 & 1.7884 \\
\hline
\end{tabular}




\section{EULER-BERNOULLI BEAM THEORY USING THE FINITE DIFFERENCE METHOD}

\subsubsection{Free vibration analysis of a fixed-fixed beam}

The natural frequencies of a fixed-fixed beam were determined. The analysis was conducted with $n=9,13$, and 17 grid points. Then the results were extrapolated to obtain those for infinite grid points. Details of the analysis and results are listed in the supplementary file "vibration analysis of a fixed-fixed beam.” The coefficients $\lambda$ (Equation (43c)) are displayed in Table 8 below.

Table 8 Coefficients $\lambda$ of natural frequencies (first mode) of a fixed-fixed beam

\begin{tabular}{|c|c|c|c|c|}
\hline CBT & FDM & FDM & FDM & $\overline{F D M}$ \\
\hline (exact results) & Nine-point grid & Thirteen-point grid & Seventeen-point grid & $\mathrm{n}=\infty$ \\
\hline 22.40 & 22.00 & 22.21 & 22.28 & 22.43 \\
\hline
\end{tabular}

The results of the present study have a high accuracy.

\subsubsection{Free vibration analysis of a tapered free-fixed beam}

The natural frequencies of the tapered free-fixed beam represented in Figure 24 were determined.

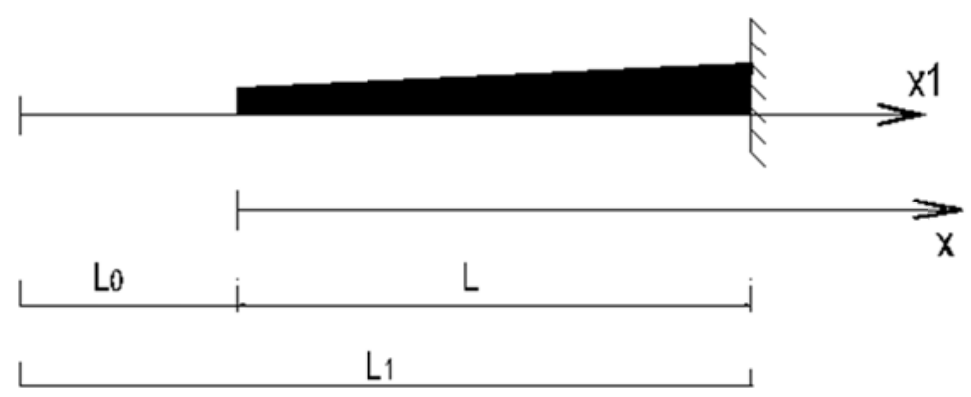

Figure 24 Vibration analysis of a tapered free-fixed beam

At a position $\mathrm{x}_{1}$ of the beam, the second moment of area $\mathrm{I}\left(\mathrm{x}_{1}\right)$ is defined similarly to Equation (55). The cross-sectional area $\mathrm{A}\left(\mathrm{x}_{1}\right)$ and the parameter $\beta_{\mathrm{A}}(\mathrm{x})$ (Equation (43c)) are defined as follows:

$$
A\left(x_{1}\right)=A_{1}\left(x_{1} / L_{1}\right)^{2} \quad \text { (58) } \quad \beta_{A}(x)=\left(x_{1} / L_{1}\right)^{2}
$$

$\mathrm{A}_{1}$ being the cross-sectional area at $\mathrm{x}_{1}=\mathrm{L}_{1}$. The analysis was conducted using the M-W FDM and W FDM approximations with $n=9,13$, and 17 grid points. Due to the low rate of convergence of the W FDM approximation, the calculation was extended to $n=25,33,49$, and 65 grid points. The analysis and results are detailed in the supplementary file "vibration analysis of a tapered free-fixed beam."

The vibration frequency $\omega$ is defined as follows (definition adopted from Torabi [3]).

$$
\omega=\lambda_{T}^{2} \times \sqrt{\frac{E I_{1}}{\rho A_{1} l^{4}}}
$$


Table 9 lists the results obtained by Torabi [3] and those obtained in the present study.

Table 9 Coefficients $\boldsymbol{\lambda}_{\mathrm{T}}$ of natural frequencies (first mode) of a tapered beam

\begin{tabular}{lccccccc}
\hline & Torabi [3] & \multicolumn{2}{c}{ Nine-point grid } & \multicolumn{2}{c}{ Thirteen-point grid } & \multicolumn{2}{c}{ Seventeen-point grid } \\
& & M-W FDM & W FDM & M-W FDM & W FDM & M-W FDM & W FDM \\
\hline$\xi_{0}=0.10$ & 2.6842 & 2.7100 & 2.4954 & 2.6957 & 2.6100 & 2.6906 & 2.6450 \\
$\xi_{0}=0.30$ & 2.3471 & 2.3548 & 2.2331 & 2.3506 & 2.2925 & 2.3491 & 2.3156 \\
$\xi_{0}=0.50$ & 2.1504 & 2.1493 & 2.0766 & 2.1500 & 2.0776 & 2.1503 & 2.1311 \\
$\xi_{0}=0.70$ & 2.0165 & 2.0101 & 1.9762 & 2.0137 & 1.9983 & 2.0135 & 2.0062 \\
$\xi_{0}=0.90$ & 1.9166 & 1.9062 & 1.8995 & 1.9120 & 1.9090 & 1.9157 & 1.9123 \\
\hline
\end{tabular}

\begin{tabular}{lccccc}
\hline & Torabi [3] & 25-point grid & 33-point grid & 49-point grid & 65-point grid \\
& & W FDM & W FDM & W FDM & W FDM \\
\hline$\xi_{0}=0.10$ & 2.6842 & 2.6675 & 2.6750 & 2.6801 & 2.6819 \\
$\xi_{0}=0.30$ & 2.3471 & 2.3329 & 2.3391 & 2.3436 & 2.3451 \\
$\xi_{0}=0.50$ & 2.1504 & 2.1419 & 2.1457 & 2.1484 & 2.1494 \\
$\xi_{0}=0.70$ & 2.0165 & 2.0120 & 2.0140 & 2.0155 & 2.0160 \\
$\xi_{0}=0.90$ & 1.9166 & 1.9147 & 1.9156 & 1.9162 & 1.9164 \\
\hline
\end{tabular}

The results of the present study are in good agreement with those presented by Torabi [3]. The M-W FDM approximation converges faster towards the exact results than the W FDM approximation.

\section{Conclusions}

The FDM-based model developed in this paper enabled, with relative easiness, first-order analysis, second-order analysis, and vibration analysis of Euler-Bernoulli beams. The results showed that the calculations conducted as described in this paper were accurate; especially in first-order analysis of uniform beams, the results were exact for uniformly distributed and concentrated loads regardless of the grid. First- and second-order element stiffness matrices (the axial force being tensile or compressive) in local coordinates were determined. Tapered beams were also analyzed. The following aspects were not addressed in this study but could be analyzed with the model in future research: 
Polynomial refinement (fifth-order polynomial, sixth-order polynomial ...) for the derivation of finite difference approximations

$\checkmark \quad$ Analysis of linear structures, such as frames, through the transformation of element stiffness matrices from local coordinates in the global coordinates.

$\checkmark \quad$ Second-order analysis of frames free to sidesway, the P- $\Delta$ effect being examined.

$\checkmark \quad$ Euler-Bernoulli beams resting on Pasternak foundations.

$\checkmark \quad$ Elastically connected multiple-beam system.

$\checkmark \quad$ Warping torsion of beams, lateral torsional buckling.

$\checkmark \quad$ Classical plate theory (introduction of additional points at plate edges).

$\checkmark \quad$ Boundary value problem, initial value problem.

$\checkmark \quad$ Linear ordinary differential equation with constants or variable coefficients.

\section{Limitations of the study}

Large deformation theory

Supplementary Materials: The following files are uploaded during submission:

- “fixed-pinned beam subjected to a uniformly distributed load”

- " cfixed-pinned beam subjected to a concentrated load"

- “fixed-pinned beam subjected to a linearly distributed load"

- " ctapered pinned-fixed beam subjected to a uniformly distributed load"

- " “fixed-free beam subjected to a uniformly distributed load and compressive force"

- "stability of a fixed-pinned beam"

- "stability of a tapered beam"

- "vibration analysis of a fixed-fixed beam”

- "vibration analysis of a tapered free-fixed beam."

\section{Author Contributions:}

\section{Funding:}

\section{Acknowledgments:}

Conflicts of Interest: The author declares no conflict of interest. 


\section{Appendix A: $\quad$ Tapered pinned-fixed beam subjected to a uniformly distributed load}

Force method of the classical beam theory: The tapered beam (Figure 21) subjected to a uniformly distributed load was analyzed with the force method of the classical beam theory. The bending moment at the fixed-end was the redundant effort.

In the associated statically determinate system, $\mathrm{M}_{0}(\mathrm{x})$ and $\mathrm{m}(\mathrm{x})$ are the bending moments due to the distributed load and to the virtual unit moment at the fixed-end, respectively.

Let us introduce the dimensionless ordinate $\xi=\mathrm{x} / \mathrm{l}$ and $\xi_{0}=\mathrm{L}_{0} / \mathrm{L}_{1}$.

$\mathrm{M}_{0}(\mathrm{x}), \mathrm{m}(\mathrm{x})$, and $\mathrm{I}(\mathrm{x})$ can be expressed as follows

$$
\begin{aligned}
& M_{0}(x)=p x(l-x) / 2=p l^{2} \xi(1-\xi) / 2 \\
& m(x)=x / l=\xi \\
& I(x)=I_{1}\left(x_{1} / L_{1}\right)^{4}=I_{1}\left[\xi_{0}+\xi\left(1-\xi_{0}\right)\right]^{4}
\end{aligned}
$$

The bending moment $\mathrm{M}_{1}$ at the fixed end is the solution of the following equations:

$$
\begin{aligned}
& \delta_{10}=\int_{0}^{l} \frac{M_{0}(x) \times m(x)}{E I(x)} d x=\frac{p l^{3}}{E I_{1}} \times \int_{0}^{1} \frac{\xi^{2}(1-\xi)}{2\left[\xi_{0}+\xi\left(1-\xi_{0}\right)\right]^{4}} d \xi \\
& \delta_{11}=\int_{0}^{l} \frac{m(x) \times m(x)}{E I(x)} d x=\frac{l}{E I_{1}} \times \int_{0}^{1} \frac{\xi^{2}}{\left[\xi_{0}+\xi\left(1-\xi_{0}\right)\right]^{4}} d \xi \\
& M_{1}=-\frac{\delta_{10}}{\delta_{11}}
\end{aligned}
$$

Equations (A2) and (A3) were solved numerically.

The combination of Equations (A1) and (A4) yields the bending moment at any position $\mathrm{x}$, as follows:

$$
M(x)=M_{0}(x)+M_{1} \times m(x)
$$

W FDM approximation: Applying Equations (A1) and (15g-j) yields the parameters $\beta_{\mathrm{I}}(\mathrm{x}), \beta_{\mathrm{I}}^{\prime}(\mathrm{x})$, and $\beta_{\mathrm{I}}^{\prime \prime}(\mathrm{x})$ as follows:

$$
\begin{aligned}
& \beta_{I}(x)=\left[\xi_{0}+\frac{x}{l}\left(1-\xi_{0}\right)\right]^{4} \\
& \beta_{I}^{\prime}(x)=4 \beta_{l k}\left(1-\xi_{0}\right)\left[\xi_{0}+\frac{x}{l}\left(1-\xi_{0}\right)\right]^{3} \\
& \beta_{I}^{\prime \prime}(x)=12 \beta_{l k}^{2}\left(1-\xi_{0}\right)^{2}\left[\xi_{0}+\frac{x}{l}\left(1-\xi_{0}\right)\right]^{2}
\end{aligned}
$$




\section{EULER-BERNOULLI BEAM THEORY USING THE FINITE DIFFERENCE METHOD}

\section{References}

[1] E. F. Anley, Z. Zheng. Finite Difference Method for Two-Sided Two Dimensional Space Fractional ConvectionDiffusion Problem with Source Term. Mathematics 2020, 8, 1878; https://doi.org/10.3390/math8111878

[2] M. Kindelan, M. Moscoso, P. Gonzalez-Rodriguez. Optimized Finite Difference Formulas for Accurate High Frequency Components. Mathematical Problems in Engineering. Volume 2016, Article ID 7860618, 15 pages. http://dx.doi.org/10.1155/2016/7860618

[3] K. Torabi, H. Afshari, M. Sadeghi, H. Toghian. Exact Closed-Form Solution for Vibration Analysis of Truncated Conical and Tapered Beams Carrying Multiple Concentrated Masses. Journal of Solid Mechanics Vol. 9, No. 4 (2017) pp. 760-782

[4] J.T. Katsikadelis. A New Direct Time Integration Method for the Equations of Motion in Structural Dynamics. Conference Paper · July 2011. Third Serbian (28th Yu) Congress on Theoretical and Applied Mechanics. https://www.researchgate.net/publication/280154205

[5] M. Soltani, A. Sistani, B. Asgarian. Free Vibration Analysis of Beams with Variable Flexural Rigidity Resting on one or two Parameter Elastic Foundations using Finite Difference Method. Conference Paper. The 2016 World Congress on The 2016 Structures Congress (Structures16)

[6] S. Boreyri, P. Mohtat, M. J. Ketabdari, A.Moosavi. Vibration analysis of a tapered beam with exponentially varying thickness resting on Winkler foundation using the differential transform method. International Journal of Physical Research, 2 (1) (2014) 10-15. doi: 10.14419/ijpr.v2i1.2152

[7] K. O. Mwabora, J. K. Sigey, J. A. Okelo, K. Giterere. A numerical study on transverse vibration of Euler-Bernoulli beam. IJESIT, Volume 8, Issue 3, May 2019 